\title{
CAMA
}

Centre for Applied Macroeconomic Analysis

\section{Uncertainty, rationality and complexity in a multi sectoral dynamic model: the Dynamic Stochastic Generalized Aggregation approach}

\section{CAMA Working Paper 16/2016 April 2016}

\section{Michele Catalano}

Prometeia, Italy

\section{Corrado Di Guilmi}

Economics Discipline Group - University of Technology Sydney and

Centre for Applied Macroeconomic Analysis, ANU

\section{Abstract}

The paper proposes an innovative approach for the analytical solution of agent-based models. The approach is termed Dynamic Stochastic Generalized Aggregation (DSG-A) and is tested on a macroeconomic model articulated in a job and in a goods markets with a large number of heterogeneous and interacting agents (namely firms and workers). The agents heuristically adapt their expectations by interpreting the signals from the market and give rise to macroeconomic regularities. The model is analytically solved in two different scenarios. In the first, the emergent proper- ties of the system are determined uniquely by the myopic behavior of the agents while, in the second, a social planner quantifies the optimal number of agents adopting a particular strategy. The integration of the DSG-A approach with intertemporal optimal control allows the identification of multiple equilibria and their qualitative classification. 


\title{
Keywords
}

aggregation, uncertainty, opinion dynamics, master equation, optimal control

\author{
JEL Classification
}

C61,E03,E32

Address for correspondence:

(E) cama.admin@anu.edu.au

ISSN 2206-0332

The Centre for Applied Macroeconomic Analysis in the Crawford School of Public Policy has been established to build strong links between professional macroeconomists. It provides a forum for quality macroeconomic research and discussion of policy issues between academia, government and the private sector.

The Crawford School of Public Policy is the Australian National University's public policy school, serving and influencing Australia, Asia and the Pacific through advanced policy research, graduate and executive education, and policy impact. 


\title{
Uncertainty, rationality and complexity in a multi-sectoral dynamic model: the Dynamic Stochastic Generalized Aggregation approach
}

\author{
Michele Catalano $^{1}$ and Corrado Di Guilmi ${ }^{2,3}$ \\ ${ }^{1}$ Prometeia - Bologna, Italy \\ ${ }^{2}$ Economics Discipline Group - University of Technology Sydney \\ ${ }^{3}$ Centre for Applied Macroeconomic Analysis - Australian National \\ University
}

March 29, 2016

\begin{abstract}
The paper proposes an innovative approach for the analytical solution of agent-based models. The approach is termed Dynamic Stochastic Generalized Aggregation (DSG-A) and is tested on a macroeconomic model articulated in a job and in a goods markets with a large number of heterogeneous and interacting agents (namely firms and workers). The agents heuristically adapt their expectations by interpreting the signals from the market and give rise to macroeconomic regularities. The model is analytically solved in two different scenarios. In the first, the emergent properties of the system are determined uniquely by the myopic behavior of the agents while, in the second, a social planner quantifies the optimal number of agents adopting a particular strategy. The integration of the DSG-A approach with intertemporal optimal control allows the identification of multiple equilibria and their qualitative classification.
\end{abstract}

Keywords: aggregation, uncertainty, opinion dynamics, master equation, optimal control.

JEL codes: C61,E03,E32.

Acknowledgments: We thank Mauro Gallegati, David Goldbaum, Simone Landini, the participants to the WEHIA and CEF conferences 2015 for valuable comments. Financial support by the Business School of the University of Technology Sydney is gratefully acknowledged. 


\section{Introduction}

The standard assumption of perfect rationality rules out the possibility of agents making mutually inconsistent decisions that may lead to situations of aggregate disequilibrium, multiple equilibria or indeterminacy. These outcomes can emerge in models that feature some sort of bounded rationality or heterogeneous beliefs (not simply constrained into a known distribution) and that allow for agents' interaction and learning. Agent-based models (ABMs) represent a suitable and well-known example of this modeling strategy. This approach proved to be able to replicate a wide range of stylized facts (see Delli Gatti et al., 2005; Dosi et al., 2013; Lengnick, 2013, among many others) and to provide original policy indications (Journal of Economic Behavior \& Organization, 2008). The Dynamic Stochastic General Equilibrium (DSGE) and the ABM approaches appear therefore at odd with respect to their conceptual pillars and the range of possible implications. While it is possible to find examples of DSGE models that incorporate some of the ABMs' insights and modeling strategies ${ }^{1}$, to the best of our knowledge the literature has not yet provided an original theoretical framework flexible enough to simultaneously include the defining features of both approaches.

Recent developments and the applications of statistical mechanics tools in macroeconomics can open new perspectives in this direction. ${ }^{2}$ In this literature, the macroeconomic system is structured as a continuum of states, each of them corresponding to a discrete value of a state quantity, such as production or price levels. Microeconomic agents are classified into a grid of states (such as production levels, pricing strategies adopted, leverage ratio, etc...) and stochastically switch across them according to probability laws defined by the transition rates. Each macroeconomic state can be associated to different configurations at the micro-level. Since the evolution of the aggregate quantities depends on how the agents spread over the micro-states, it is then possible to quantify a probability for the macro-states. This method can work with any amount of available information about the possible microeconomic configurations.

This analytical representation has proved to be able to replicate the results of agent-based models with higher degree of heterogeneity (Chiarella and Di Guilmi, 2011; Di Guilmi et al., 2012; Di Guilmi and Carvalho, 2015) and can be therefore confidently applied in order to analytically solve this class of models.

This method is here applied to an ABM adapted from Russo et al. (2007) to provide two main contributions. First, the paper introduces an original use of the master equation in economics and finance, applying it to a set of mi-

\footnotetext{
${ }^{1}$ See Gobbi and Grazzini (2015) and the papers reviewed by Dilaver et al. (2016). From this perspective the works by Per Krusell and co-authors are also relevant (see in particular Krusell et al., 2012).

${ }^{2}$ See Alfarano et al. (2008); Aoki (1996, 2002); Aoki and Yoshikawa (2006); Foley (1994); Lux (1995, 1998); Smith and Foley (2008); Weidlich (2000, 2008). In this paper we will refer in particular to the seminal contributions by Aoki (2002) and Aoki and Yoshikawa (2006) and the further developments by Di Guilmi (2008) and Landini and Uberti (2008).
} 
croeconomic strategies. This represents a relevant contribution to the vast and growing literature about opinion dynamics (see Brock and Hommes, 1997; Lux, 2009, among many others) from a twofold perspective: first, the model is explicitly microfounded and, second, a closed form solution is identified, allowing for a full analytical representation of the evolution of the macro-variables as dependent on agents' choices. The aggregate model preserves the behavioral assumptions of the ABM by embodying them in the transition rates. Consequently, the aggregated system inherits the disequilibrium dynamics from the ABM model. For this reason, a side result is that our analytical model can be regarded as an original alternative to model dynamical disequilibrium. We define this innovative approach as Dynamic Stochastic Generalized Aggregation $(D S G-A)$.

The second contribution is in that the DSG-A approach is able to incorporate infinite horizon optimization in a complex economic system, allowing for a comparison with the heuristic behavior of the standard ABM treatment. As a result, we are able to model and discriminate between: a) rational incentives driving microeconomic behavior and, via aggregation, the macroeconomic dynamics; b) the uncertainty arising from irreducible complexity due to agents' interaction. This representation involves interaction at the microeconomic level and it could not be reproduced by standard stochastic processes nor be included in a standard decision process under uncertainty. This allows us not only to identify a multiple-equilibria system but also to qualitatively distinguish different types of equilibria. Namely two kinds of equilibria emerge: the rational equilibrium, where the system lies in a quasi-steady state defined by rationality principles, and the uncertainty equilibrium, which is the result of the system complexity and agents' interaction. In the rational equilibrium the agents receive signals that lead the macroeconomic system to stabilize, according to an optimization rule identified by a social planner. The uncertainty equilibrium corresponds to a sub-optimal configuration determined by the uncoordinated response of agents to changes in the economic environment.

Anticipating some of the results, the analysis shows that full employment equilibria is attainable only in a context with perfect rationality. In both the heuristic and the optimizing treatments, the model exhibits structural imbalances that lead to periodic crashes when agents are boundedly rational or affect the longrun trend in the other case. Even in the case of perfect rationality, the economy can be caught in an uncertainty trap (Aoki and Yoshikawa, 2006, chap. 4).

The remainder of the paper is structured as follows. Section 2 introduces in very general terms the methodology of aggregation and, in particular, the concept of statistical entropy and master equation. This section also presents the two treatments of the stochastic evolution of the agents: the heuristic (or zerointelligence) case and the full rationality case. Section 3 briefly presents the model while section 4 shows how the assumptions of the model are embodied into the analytical solution. Section 5 illustrates and contrasts the results of the numerical simulations for the the heuristic case and the full rationality case. 
Section 6 analytically identifies the different types of equilibria and the transition paths, presenting also a simple policy experiment. Finally section 7 offers some concluding remarks.

\section{Methodology}

This section presents the main tools used by the DSG-A approach, in particular statistical entropy (subsection 2.1) and master equation (subsection 2.2). Subsection 2.3 shows how different degrees of rationality and uncertainty are incorporated into the transition rates in order to determine the stochastic evolution of the system.

\section{$2.1 \quad$ Entropy and inference}

As the economy is populated by a very large number of dissimilar agents, an analytical model cannot keep track of the conditions of every single agent at each point in time. Therefore, a model should consider how many agents are in a certain condition rather than which agents. The different possible conditions of the microeconomic agents can be defined as micro-states. Assuming the state of the macroeconomy to be dependent on the relative densities of agents in each micro-state, it is possible to map the different configurations at the micro-level into a given macroeconomic outcome. Consequently, the statistical distribution of the agents can provide a probabilistic description of the evolution of the macroeconomy. Maximizing the number of ways in which a particular macro configuration can be obtained, we can identify the most likely dynamics of the macroeconomy given our information on the micro-states.

The maximization of the system entropy, known in information theory as MaxEnt (Jaynes, 1957), provides the most likely probability function for the number of agents following a particular behavioral rule $s$, subject to the normalization constraint and to additional constraints representing the available information. As demonstrated in A, the resulting functional form for the probability distribution is given by

$$
\operatorname{Prob}\left(n_{s}\right)=\frac{e^{-\beta V_{s}}}{\sum_{s} e^{-\beta V_{s}}},
$$

where $V_{s}$ is the payoff associated to the $s^{\text {th }}$ strategy. The quantity $\beta$ is the intensity of switching and measures the degree of uncertainty in the system: for $\beta \rightarrow$ $\infty$ there is no uncertainty and all agents follows the best-performing strategy; for $\beta \rightarrow 0$ the payoff plays no role and all the strategies have equal probability. This functional form has become extremely popular in discrete choice model since the pioneering work by Brock and Hommes (1997). Chiarella and Di Guilmi (2014) show how this formulation can be endogenously derived by maximizing the statistical entropy. In this approach, $\beta$ is the Langrange multiplier of the constraint to the entropy maximization concerning the returns coming from the different 
states, rather than an exogenous parameter. As shown in the appendix, in the case of two available strategies 1 and $2, \beta$ can be expressed as

$$
\beta=\left(V_{1}-V_{2}\right)^{-1} \log \left(\frac{n_{1}}{1-n_{1}}\right) .
$$

It is easy to see that, provided that $V_{1}-V_{2} \neq 0$ and is finite, $\beta \rightarrow \pm \infty$ when $n_{1}$ goes to 0 or 1 (minimum uncertainty: all agents adopt the same strategy), while $\beta=0$ when $n_{1}=1 / 2$ (maximum uncertainty: the two strategies are equally likely).

\subsection{Master Equation and dynamics}

Consider a population of $i=\left[1, \ldots, N_{k}\right]$ agents, where $k$ identifies the subgroup, for example firms or workers. At any point in time, an agent is in a state $s_{k}=$ $\left[1, \ldots, S_{k}\right]$ and adopts the associated strategy $f_{s_{k}}$ in order to set the evolution if its control variables $\mathbf{x}_{k} \in R^{M_{k}}$, where $M_{k}$ is the number of control variables for each type of agent. Thus the control variables of the individual $i$ of type $k$ evolves according to the equations $\dot{x}_{i, k}=f_{s_{k}}\left(x_{i, k}\right)$. For the whole economy, it is possible to identify a $S \times m_{k}$ functional matrix $F$ and a $m_{k}$-dimensional control variable vector $\dot{\mathrm{x}}$ such that

$$
\dot{\mathbf{x}}(t)=F(\mathbf{x}(t)) .
$$

In order to reduce the dimension of the problem and aggregate the system, let us indicate the probability of an agent to be in state $s_{k}$ as $P\left(s_{k}\right)$. The resulting aggregated dynamical system is:

$$
\dot{X}_{k}=\sum_{s_{k}} f_{s_{k}}\left(X_{k}\right) P\left(s_{k}\right)
$$

where $\dot{X}_{k}$ is the variation of the aggregate control variable. The above system is the aggregated stochastic macro-rule originating from the set of micro-rules (3).

Assuming that the stochastic process governing the switching of a specific $\mathrm{j}$-type agent is of the Markovian type ${ }^{3}$ we can use the master equation to model the evolution of the probabilities $P(s)$. Considering only two states $s=[1,2]$ and dropping the subscript $k$ to simplify the notation, the master equation for the density $n_{s}$ of agents in state $s$ is given by

$$
\frac{d P\left(n_{s}, t\right)}{d t}=\lambda(t) P\left(n_{s}-1 / N, t\right)+\gamma(t) P\left(n_{s}+1 / N, t\right)-[\lambda(t)+\gamma(t)] P\left(n_{s}, t\right)
$$

\footnotetext{
${ }^{3}$ It is worth remarking that the transition rates of the process are time varying and, as a consequence, the assumption of Markovianity does not imply the memory-less property at the agent level. In fact, the probability of transition of an agent depends on its current endowment, which is the result of its previous history, and its current micro-state, which is updated every period. On the use of Markov processes to represent the dynamics of ABMs see Izquierdo et al. (2009).
} 
where $\lambda$ and $\gamma$ are the transition rates, respectively, in and out of state $s$. Equation (5) is a balance flow equation between the probability of observing a density equal to $n_{s}$ starting from a different density and the probability of already having a proportion $n_{s}$ of agents in state $s$ and observe any transition. The transition rates are given by

$$
\begin{aligned}
& \lambda(t)=(1-\eta) \zeta(t), \\
& \gamma(t)=\eta \iota(t) .
\end{aligned}
$$

where $\eta$ is the probability to be in state $s$, which is considered as exogenous for the moment, while $\zeta$ and $\iota$ are the probabilities for a single agent of, respectively, entering and exiting state $s$. The transition probabilities are dependent on the behavioral assumptions of the model and the agent's condition at any given time. ${ }^{4}$

In order to asymptotically solve (5), we apply the approximation method introduced by Aoki (2002), which splits the fraction of agents in state $s, n_{s}$, into a drift term $m$ and an additive spread term $u$ (divided by $N^{1 / 2}$ to normalize its standard deviation) as follows:

$$
n_{s}(t)=m(t)+u(t) N^{-1 / 2} .
$$

For the case of a binary option for agents, it is possible to derive a system of coupled equations for the generic term $m$ and $u$ :

$$
\begin{gathered}
\frac{d m}{d t}=\lambda(t) m(t)-[\lambda(t)+\gamma(t)] m(t)^{2} \\
d u=-a(m) u d t+b(m) d W
\end{gathered}
$$

where $d W$ is a Wiener process. ${ }^{5}$

The system of equations (8), (9) and (10) defines the dynamical system for the evolution of the agent distribution over the strategy set.

Accordingly, equation (4) can be re-formulated as follows

$$
\dot{X}_{k}(t)=\sum_{s_{k}} f_{s_{k}}\left(X_{k}(t)\right) n_{s_{k}}(t)
$$

\footnotetext{
${ }^{4}$ The DSG-A makes use of endogenous and microfounded transition rates as opposed to the standard approach in DSGE with heterogeneous agents, in which the heterogeneity is modeled as an idiosyncratic exogenous stochastic process.

${ }^{5}$ Di Guilmi (2008) derives a solution of the master equation (5) composed of equation (9) and a Fokker-Planck equation whose stationary solution is

$$
P(u)=C e^{-u^{2} / 2 \sigma^{2}},
$$

such that $P(u) \sim \mathcal{N}\left(0, \frac{\lambda \gamma}{(\lambda+\gamma)^{2}}\right)$. As demonstrated by Gardiner (2002) and van Kampen (1992), the stochastic process of the noise can be expressed as the Ito stochastic differential equation (10).
} 
It is finally possibly to build a dynamical system to describe the model. This system is composed by two subsystems: the subsystem of macro-equations (12), which is nothing more than a weighted average of the rules of the system at the micro level, and the subsystem of the master equations' solutions that determines the number of agents in the states according to equations (8), (9) and (10). The first subsystem uses the proportions of agents in each state to describe the time evolution of the macroeconomic variables, while the second uses the transition rates, which are updated according to the response of agents to changes in the macroeconomy, to provide the proportions of agents in the different states.

\subsection{Stochastic equilibrium}

Since we are modeling the switching dynamics as a Markov process, it is possible to identify the stationary distribution of the stochastic process in order to assess the long-term properties of the system.

\subsubsection{Equilibrium distribution}

The master equation is in equilibrium when the probability inflows are equal to the outflows. Following Aoki $(2002,44)$, in the case of two possible states $s=[1,2]$, the probability function for $n_{1}$, under this condition, is of the Gibbs type, with the following functional form ${ }^{6}$

$$
P\left(n_{1}\right)=\eta=\frac{e^{\beta g\left(n_{1}\right)}}{e^{\beta g\left(n_{1}\right)}+e^{-\beta g\left(n_{2}\right)}} .
$$

This formulation for the stationary probability allows us to incorporate additional information with respect to the result in (1). In (13), the economic behavior is not simply measured by the payoff of the strategy but is modeled by $g\left(n_{s}\right)$, which is a function evaluating the difference in the utility between the different strategies.

In the standard opinion formation literature, the function $g\left(n_{s}\right)$ in (13) represents an assumed fitness function that quantifies the returns associated to the particular strategy $s$. Alternatively, the suitable functional form for $g$ can be endogenously identified using the economic potential (Smith and Foley, 2008). The potential is a functional that quantifies the likelihood of a state of the system as a consequence of the states of its parts. From this perspective, it provides a measure of uncertainty since it depends on how many combinations of agents' choices are compatible with a given macroeconomic state. In particular, large values of the potential signals that a particular macroeconomic state can be generated by a great number of different configurations at the micro-level, therefore

\footnotetext{
${ }^{6}$ This result stems from the Markov-Gibbs equivalence demonstrated by the Hammersley and Clifford theorem (Clifford, 1990).
} 
the degree of uncertainty in the system is large. ${ }^{7}$ Consequently, the minima of the potential represent possible absorbing states where the agents have no incentive to change strategy and the discontinuities can prevent the transition from one equilibrium to another. Following Aoki and Yoshikawa (2006) and Di Guilmi (2008), for systems with two micro-states, the potential is defined as

$$
U=-2 \int_{0}^{n_{1}} g(x) d x-\frac{H\left(n_{1}\right)}{\beta},
$$

where $H$ is the statistical entropy, which in the Shannon formulation is given by

$$
H=-n_{1} \log \left(n_{1}\right)-n_{2} \log \left(n_{2}\right) .
$$

As equation (14) shows, a negative relationship exists between the level of the potential and the uncertainty, quantified by $\beta$. When the uncertainty is at its maximum $(\beta \rightarrow 0)$ the potential is not defined and tends to infinity.

\subsubsection{Agents' behavior and stochastic equilibrium}

The $g$ function plays a relevant role in our story. It factors the degree of rationality in the determination of the stochastic equilibrium. We distinguish two cases. In the first scenario, the heuristic case, the aggregate properties are the results of the uncoordinated choices of agents, who behave like atoms. In the second case, very much in line with Brock and Durlauf (2001), we identify the proportions of agents in each group that maximize some measure of social welfare by applying standard maximization tools.

\section{Derivation of $g$ : the heuristic case}

The critical points are the solutions of the first order condition of equation (14) with respect to $n_{1}$, which is defined as

$$
\frac{d U}{d n_{1}}=-2 g\left(n_{1}\right)-\frac{d H}{d n_{1}} \beta^{-1}=0 .
$$

Considering that $n_{2}=1-n_{1}$ and substituting (2) and (15) into (16), we obtain

$$
g\left(n_{1}\right)=\frac{V_{1}-V_{2}}{2} .
$$

At the point of minimum uncertainty, $g$ is equal to the relative value of the payoffs, with an equal probability for the two alternatives.

Substituting (2) and (17) into (13), the probability (13) simplifies to

$$
P\left(n_{1}\right)=\frac{e^{\beta g\left(n_{1}\right)}}{\mathcal{Z}}=\frac{e^{\frac{V_{1}-V_{2}}{2} \log \left(\frac{n_{1}}{1-n_{1}}\right)\left(V_{1}-V_{2}\right)^{-1}}}{\mathcal{Z}}=\frac{n_{1}}{1-n_{1}} \frac{1}{\mathcal{Z}},
$$

\footnotetext{
${ }^{7}$ In statistical physics, the minimum of the potential are the points in which the free energy of the system, and consequently the uncertainty, reaches a minimum. In an economy this corresponds to a situation where incentives to take opposite actions offset each other and the system reaches a statistical equilibrium.
} 
where $\mathcal{Z}=\sum_{s} P\left(n_{s}\right)$. Under the assumption of heuristic behavior, the product of the uncertainty variable $\beta$ and the $g$ function vanishes: if economic behavior is quasi-random, the economic incentives are indistinguishable due to uncertainty.

\section{Derivation of $g$ : full rationality and optimization}

Rationality is introduced in the model in order to preserve the probabilistic nature of the aggregation method, allowing for a comparison with the heuristic case. The decision rules for agents are the same as in the heuristic setting but we furthermore assume that a social planner maximizes an objective function whose arguments are the fractions of agents adopting a particular rule. In a treatment similar to Brock and Durlauf (2001), the social decision maker optimizes an intertemporal objective function controlling for the densities of agents in each group. ${ }^{8}$ From this perspective, the $g$ function becomes the outcome of a dynamic intertemporal control problem specified as marginal net utility from available alternatives. The fully rational optimization and the heuristic behavior can be regarded as the extreme cases within the same modeling approach.

The social planner considers equation (12) for the macro-state $Y$ as the state equation of a standard intertemporal control problem. The control instruments are given by the fractions of agents choosing a particular strategy.

Formally, the vector of the occupation numbers $\mathbf{n}$ quantifies the weights for a collection of available strategies $s=1, \ldots, N$. The intertemporal optimization problem is defined by the following infinite horizon payoff stream

$$
g\left(Y, n_{s}\right)=\max _{\mathbf{n}(t)} \int_{t_{0}}^{\infty} e^{-\theta t} \psi(Y(t), \mathbf{n}(t)) d t,
$$

subject to the macroeconomic rule:

$$
\dot{Y}(t)=\sum_{s} f_{s}(Y(t)) n_{s}
$$

where $\psi$ and $\theta$ are respectively the instant payoff function and the discount factor. The steps required to obtain a closed form solution for the above problem are:

1. to represent the optimization problem under the Hamilton-Jacobi-Belmann equation, which provides a dynamic forward looking ordinary differential equation for the discounted payoff function (18);

2. to calculate the first order condition with respect to $\mathbf{n}$, subject to the dynamics of the state variable given by eq. (19);

\footnotetext{
${ }^{8}$ This is a standard modeling strategy in macroeconomics, for example in the dynamic programming applied to search and matching labor market (Trigari, 2006). In these models, labor participation decision is modeled as an intertemporal problem of a planner that chooses the optimal fraction of household's working members. Optimality conditions provides dynamic labor supply equations that depends on the expected net labor income. Similarly, we use the densities of agents in each group as control variables, to be set by the social planner given some dynamic constraints, in order to maximize the intertemporal utility/profits.
} 
3. finally, to find a closed form solution for $g$, i.e. the solution in time for the dynamics of the value function found in step 2 .

In economic terms, the problem boils down to the decision about the fractions of individuals playing each of the available strategies. If applied at the individual level, such a model would describe an agent playing a mixed-strategy. From a frequentist or aggregate perspective, the solution for the $g$ function is used to describe a world where agents are guided by rationality principles while adopting simple behavioral rules. This approach to rationality does not need a reduction of the degree of complexity of the system and it is able to describe rationality in a context of disequilibrium.

\section{The Agent-Based Model}

The model on which we test the above described tools borrows heavily from Russo et al. (2007). This model provides a suitable environment for testing the DSG-A because it includes different markets in a rather simple setting with basic heuristic behavioral rules for agents.

Our model is composed by a large and close population of firms and workers and the economic system is a circular flow of goods and money. Firms operate in a monopolistically competitive market: they produce goods that are close substitutes but, given market imperfections, can be sold at different prices. Firms can be heterogeneous for level of production and price. Workers have identical skills but, given labor market frictions, they can be characterized by different reservation wages. They also can have different levels of wealth. Firms set the production quantity, the selling prices and the labor demand. Within the model information is incomplete and limited. Agents adaptively revise their expectations each period according to a set of simple rules.

Firms heuristically determine the quantity to produce depending on whether they sold the whole production in the previous unit of time or they have unsold goods in stock $S_{i}$. Namely, the $i^{\text {th }}$ firm decides to adjust the desired produced quantity $y_{i}(t)$ with a simple dichotomic strategy:

$$
y_{i}(t)=y_{i}(t-d t) \times\left\{\begin{array}{l}
(1+\delta) \text { if } S_{i}(t-d t)=0 \\
(1-\delta) \text { if } S_{i}(t-d t)>0
\end{array}\right.
$$

where $0<\delta<1$. Assuming a linear production function $y_{i}(t)=l_{i}(t)$ with the unitary labor productivity constant across firm and through time, in order to produce the desired output each firm will demand a quantity of labor equal to

$$
l_{i}(t)=y_{i}(t)
$$

Also workers adaptively revise their strategy according to the previous period's state. Workers set satisficing wages $w^{s}$ according to their previous occupation 
status, which is a dichotomic variable occ equal to 1 if the worker was employed and equal to 0 otherwise. Namely the satisficing wage of worker $j$ is equal to

$$
w_{j}^{s}(t)=w_{j}^{s}(t-d t) \times\left\{\begin{array}{l}
(1+\delta) \text { if } o c c_{j}(t-d t)=1 \\
(1-\delta) \text { if } o c c_{j}(t-d t)=0 .
\end{array}\right.
$$

As the labor market opens, firms set vacancies according to the difference between labor demand and their current stock of labor force. If the difference is positive new vacancies are to be filled, otherwise labor is destroyed. Then the $j^{\text {th }}$ worker sends applications to a subset $h_{w, j}$ of randomly chosen firms, indicating her satisficing wage. Firms collect randomly the workers curricula, then sort them by satisficing wage and hire the cheapest workers. As a consequence, a firm may not receive enough applications to satisfy its demand for new labor and the actual increase in output will be smaller than the planned $\delta y_{i}$. In the case of a firm reducing its output, the most expensive employees will be laid off.

Once the quantity of labor is set and wage costs are known, firms set the goods price for the current period. Firms are price-setters. Their decision is dependent on the fact that they were or not able to sell all their production in the previous unit of time. If a firm did not sell all its output, and thus it has accumulated stocks, it will revise the price downward. The opposite decision is taken if its stocks are equal to zero. Accordingly, the desired price $p_{i}^{*}(t)$ for firm $i s$

$$
p_{i}^{*}(t)= \begin{cases}p_{i}(t-d t)(1+\delta) & \text { if } S_{i}(t)=0 \\ p_{i}(t-d t)(1-\delta) & \text { if } S_{i}(t)>0 .\end{cases}
$$

A firm will actually apply the variation calculated as in (23) if the resulting price is at least equal to the production costs. Defining $p_{i}^{1}=\frac{\sum_{j \in h_{l, i}} w_{j}(t)}{y_{i}(t)}$ the average cost over the subset $h_{l, i}$ of employed workers, the actual price for the firm $i$ at time $t$ will be equal to

$$
p_{i}(t)= \begin{cases}p_{i}^{*}(t) & \text { if } p_{i}^{*}(t) \geq p_{i}^{1}(t) \\ p_{i}^{1}(t) & \text { if } p_{i}^{*}(t)<p_{i}^{1}(t) .\end{cases}
$$

The $j^{\text {th }}$ consumer wants to consume all her wealth. She looks for the cheapest goods collecting a subset of posted prices from $h_{j, y}$ randomly chosen firms. Then she sorts the prices and buys goods in the quantity compatible with her endowment of wealth (given the prices). As such consumers can be supplyconstrained, if the amount of goods purchased from the firms they visit is below the desired quantity. Worker's wealth $z_{j}$ is increased in each period by the amount of labor income plus a share of firms' profits (equally allocated among all households) less consumption. If the consumer is supply-constrained, the wealth in excess of consumption is remunerated at the constant interest rate $r$ in each period. In the following period she will try again to spend all her wealth. 


\section{Stochastic aggregated model}

In this section we apply the aggregation method introduced in section 2 to the model presented in section 3. Firms and workers are classified according to their strategy or condition. The densities of agents in each condition determines the evolution of the relevant macrovariables in our economy, namely production, price, wage and consumption. The dynamics of these densities are identified by a set of four master equations. Consequently, the dynamical system describing the model is composed by four aggregate equations, as (12), and the solutions of the master equations, given by (8), (9) and (10) for each of the macroeconomic variables. The solutions of the master equations feature the transition rates. These rates are specified in accordance to the behavioral rules of the $\mathrm{ABM}$ and therefore constitute the link between the microeconomic level and the macroeconomy.

The necessary steps in order to perform the aggregation are:

1. the identification of the states over the agents' sub-groups. Namely, we have $k=\{y, c, w, p\}$ for, respectively, firms clustered for production level, consumers, workers and firms clustered for price. Table 1 shows the states representing agents choices;

2. the definition of the equations for the agents' state variables according to equation (3);

3. the specification of the transition rates to plug into the master equations. In order to define the transition rates we need: first, to identify the transition probabilities and, second, to quantify the generic probability $\eta$ for an agent of being in one the two states. For the latter task we need to specify the uncertainty variable $\beta$ and the value functions $g$ for, respectively, the heuristic case and the rational case.

The remainder of this section presents the results of steps 2 and 3 while the full detail of the derivation is provided in $\mathrm{B}$ and $\mathrm{C}$.

\subsection{Aggregate equations}

In order to complete the first step we need to make use of the law of motion of the variables at the micro-level defined in the previous section and then aggregate by calculating a suitable weighted average with the weights given by the proportion of agents in each the two states, determined by the master equation.

The equation governing the dynamics of aggregate production is the weighted mean of the variation in production for firms that reduce production and firms that increase production to an extent dependent on the matching the labor market. 
To analytically approximate the imperfect matching generated by the random procedure of the ABM, we use the matching function $f_{l}\left(u_{n}(t), y_{i}(t) \delta\right)=$ $u_{n}(t)^{a}\left(y_{i}(t) \delta\right)^{b}$ and the associated per-vacancy matching probability $q(t)=$ $\frac{f_{l}(t)}{y_{i}(t) \delta}$ with $u_{n}(t)$ and $y_{i}(t) \delta$ denoting, respectively, the unemployed workers and the new vacancies. As a consequence, for the analytical treatment, equation (20) is re-expressed as

$$
y_{i}(t)=y_{i}(t-d t) \begin{cases}\left(1+f_{l}(t)\right) & \text { if } S_{i}(t-d t)=0 \\ \left(1-y_{i}(t) \delta\right) & \text { if } S_{i}(t-d t)>0\end{cases}
$$

Using (25), as detailed in $\mathrm{C}$, the aggregate equation can be written as

$$
\dot{Y}(t)=Y(t) \delta[1+q(t)]\left[n_{y}(t)-\frac{1}{1+q(t)}\right] .
$$

With reference to the price decision, firms can be in two possible situations: a fraction of $n_{p}$ firms decide about the price according to the rule (23); the others $\left(1-n_{p}\right)$ firms are cost constrained and therefore adopt the price $p_{i}^{1}$. Considering that the sign of price adjustment is the same as quantity adjustment since both decision depends on the presence of inventories, the average change in price will be equal to

$$
\dot{P}(t)=n_{p}(t)(\operatorname{sign}(\dot{Y}) P(t) \delta)+\left(1-n_{p}(t)\right)(\dot{W}(t)+W(t)-P(t)),
$$

which can be rewritten as

$$
\dot{P}(t)=n_{p}(t)(\operatorname{sign}(\dot{Y}) P(t) \delta+P(t)-W(t)-\dot{W}(t))+\dot{W}(t)+W(t)-P(t) .
$$

If $n_{p} \rightarrow 1$ the price adjustment follows the same procedure as quantity, if $n_{p} \rightarrow 0$ the aggregate price grows as much as wages do.

For the workers' decision about satisficing wage, given (22), the evolution of the average wage can be written as

$$
\dot{W}^{s}(t)=2 W^{s}(t) \delta\left(n_{w}(t)-\frac{1}{2}\right) .
$$

Finally, consumers can be classified in two states according to their demand level: for a fraction $n_{c}$ of consumers, demand is set simply as the real wealth; the others set their demand equal to firm's supply because constrained. Accordingly, the aggregate equation is:

$$
C(t)=n_{c}(t) Z(t)+\left(1-n_{c}(t)\right) \frac{P(t) Y(t)}{N_{z}(t)},
$$

where $Z$ is the wealth and $N_{z}$ the number of consumers/workers. Equation (29) states that aggregate consumption is the weighted mean between aggregate wealth and aggregate supply. If $n_{c}$ goes to zero we have a fully supplyconstrained market. The model in aggregate is closed for the equation governing 
the evolution of consumers wealth. Defining the number of jobless workers as $1-\frac{Y(t)}{N_{z}(t)}$ we have

$$
\dot{Z}(t)=[r Z(t)+W(t)-C(t)] \frac{Y(t)}{N_{z}(t)}+[r Z(t)-C(t)]\left(1-\frac{Y(t)}{N_{z}(t)}\right) .
$$

The first term in equation (30) is the wealth equation if workers are employed. The second term is different as wage earning is assumed to be null if workers are unemployed.

\subsection{Transition rates}

The transition rates are needed to define the four master equations (one for each alternative for firms, workers and consumers), according to (5). The transition rates are the product of the transition probability times the probability of being in the relevant state. The transition probabilities are developed according to the underlying behavioral assumptions of the model specified in section 3 and detailed in B. The generic probability of being in a given state $\eta$ is determined according to (13), for which we need to the determine the uncertainty variable $\beta$ and the value function $g$ for the heuristic case and for the rational optimization case.

\subsubsection{Heuristic behavior}

The quantity $\beta$ is computed according to equation (2) while $g$, in the heuristic treatment, according to equation (17). Using the payoffs derived in B, with some simple algebraic manipulations, we obtain: for firms' choice about output:

$$
\begin{gathered}
\beta_{y}=-\left(u_{n}(t)^{a}(Y(t) \delta)^{b}+Y(t) \delta\right)^{-1} \log \left(\frac{n_{y}(t)}{1-n_{y}(t)}\right) \\
g_{y}=\frac{1}{2}\left(u_{n}(t)^{a}(Y(t) \delta)^{b}+Y(t) \delta\right)
\end{gathered}
$$

for the dynamics of price:

$$
\begin{gathered}
\beta_{p}=-(\operatorname{sign}(\dot{Y}(t)) P(t) \delta+P(t)-W(t)-\dot{W}(t))^{-1} \log \left(\frac{n_{p}(t)}{1-n_{p}(t)}\right) \\
g_{p}=\frac{1}{2}(\dot{W}(t)+W(t)-P(t)-\operatorname{sign}(\dot{Y}(t)) P(t) \delta)
\end{gathered}
$$

for the satisficing wage for workers:

$$
\beta_{w}=-(2 W(t) \delta)^{-1} \log \left(\frac{n_{w}(t)}{1-n_{w}(t)}\right)
$$




$$
g_{w}=W(t) \delta
$$

for consumers:

$$
\begin{gathered}
\beta_{c}(t)=\left(\frac{P(t) Y(t)}{N_{z}(t)}-Z(t)\right)^{-1} \log \left(\frac{n_{c}(t)}{1-n_{c}(t)}\right), \\
g_{c}(t)=\frac{1}{2}\left(\frac{P(t) Y(t)}{N_{z}(t)}-Z(t)\right) .
\end{gathered}
$$

In the case of consumption, $\beta_{c}$ tends to infinity (minimum uncertainty) when supply and demand are in equilibrium, that is $\frac{P Y}{N_{z}}-Z=0$. As shown below, a particularly interesting situation arises when, in such a situation of goods market equilibrium, $n_{c} \approx 1 / 2$ pushing $\beta$ to 0 (maximum uncertainty). In this case numerical simulations can determine which of the two effects dominate.

\subsubsection{Rational optimization}

The optimal control is adopted for the real variables (firms' supply and households' consumption decisions) while price and wage setting are determined by heuristic behavior. As shown below, this treatment sheds light to the outcome when also the nominal variables are optimized. Since the stationary fraction of agents choosing one strategy is determined by the stationary probability (13), the social planner must solve a suitable $g\left(n_{1}\right)$ function, as to maximize the infinite horizon of expected stream of profits and utility. The problem involves the allocation of agents over two different populations (firms and workers). Consequently, the goal of the social planner is to maximize the stream of expected firms' profits and expected households' life-time utility subject, respectively, to equation (26) and (30). As per equations (18) and (19), such objective functions define the $g$ functions for, respectively, quantity-setting firms and consumers:

$$
\begin{gathered}
g_{y}=J(Y)=\max _{n_{y}} \int_{0}^{\infty} e^{-\theta t}\left[n_{y}(t)(-k)+\left(1-n_{y}(t)\right)\left(\frac{W(t)}{P(t)}-1\right)\right] d t, \\
g_{c}=J(Z)=\max _{n_{c}} \int_{0}^{\infty} e^{-\theta t}\left[n_{c}(t) u(Z(t))+\left(1-n_{c}(t)\right) u(Y(t))\right] d t .
\end{gathered}
$$

where $\theta$ is the household's discount parameter. In the case of firms, $1-\frac{W(t)}{P(t)}$ are unitary profits and $k$ is a searching cost. In the household problem, $u(Z)$ and $u(Y)$ denote the current utility deriving from the available wealth and consumption of firms' production. We also define $n_{y}(t)(-k)$ as the marginal search cost coming from the increase in production and $\left(1-n_{y}(t)\right)\left(\frac{W(t)}{P(t)}-1\right)$ as the profit loss due to a decrease in production. In the same way, $n_{c} u(Z)+\left(1-n_{c}\right) u(Y)$ 
represent the net expected utility value given from current endowment, for not supply-constrained consumers, and production levels, for supply-constrained consumers.

We then apply dynamic programming to equations (39) and (40). The corresponding Hamilton-Jacobi-Bellman equations are given by

$$
\begin{aligned}
& -J(Y, t)=\max _{n_{y}} e^{-\theta t}\left[n_{y}(t)(-k)+\left(1-n_{y}(t)\right)\left(\frac{W(t)}{P(t)}-1\right)\right]+J_{y}(Y, t) \dot{Y}(t) . \\
& -J(Z, t)=\max _{n_{c}} e^{-\theta t}\left[n_{c}(t) u(Z(t))+\left(1-n_{c}(t)\right) u(Y(t))\right]+J_{z}(Z, t) \dot{Z}(t) . \quad(42)
\end{aligned}
$$

We guess the solutions $J(Y, t)=e^{-\theta t} V(Y)$ and $J(Z, t)=e^{-\theta t} V(Z)$. Observing that $J_{Y}(Y, t)=V_{Y}(Y)$ and $J_{Z}(Z, t)=V_{Z}(Z)$, the conditions become ${ }^{9}$

$$
\begin{aligned}
-V(Y) e^{-\theta t} e^{-\theta t} & =\max _{n_{y}}\left[n_{y}(t)(-k)+\left(1-n_{y}(t)\right)\left(\frac{W(t)}{P(t)}-1\right)\right]+ \\
& +V_{y}(Y) e^{\theta t} e^{-\theta t} \dot{Y}(t) \\
-V(Z) e^{\theta t} e^{-\theta t} & =\max _{n_{c}} e^{-\theta t}\left[n_{c} u(Z(t))+\left(1-n_{c}\right) u(Y(t))\right]+ \\
& +V_{Z}(Z) e^{-\theta t} e^{-\theta t} \dot{Z}
\end{aligned}
$$

The result is an ordinary differential equation in the state variable. Substituting the dynamical constraints (26) and (30) into respectively (43) and (44) and taking the partial derivatives for $n_{y}$ and $n_{c}$, the first order conditions can be expressed as

$$
\begin{gathered}
{\left[(-k)+1-\frac{W(t)}{P(t)}\right]+J_{Y}(Y) Y(t) \delta=0,} \\
u(Z(t))-u(Y(t))+J_{Z}(Z(t)-Y(t))=0 .
\end{gathered}
$$

Substituting equations (45) and (46) into (41) and (42) for $J_{Y}$ and $J_{Z}$, and assuming $u(X)=X$, we get two ordinary differential equations in the macro

\footnotetext{
${ }^{9}$ To verify this result, consider the dynamic programming equation in discrete time$$
J\left(x_{t}, t\right)=\max _{y_{t}} U\left(x_{t}, y_{t}, t\right)+J\left(x_{t+1}, t+1\right),
$$

subject to

$$
x_{t+1}=h\left(x_{t}, y_{t}, t\right),
$$

given $x_{0}$. The expansion of the value function in $\Delta t$ gives

$$
J\left(x_{t}, t\right)=\max _{y_{t}} U\left(x_{t}, y_{t}, t\right) \delta t+J\left(x_{t}, t\right)+\frac{\partial J\left(x_{t}, t\right)}{\partial x_{t}} \frac{x_{t+\Delta t}-x_{t}}{\Delta t} \Delta t+\frac{\partial J\left(x_{t}, t\right)}{\partial t} \Delta t+o(\Delta t) .
$$
}

Taking the limit $\Delta_{t} \rightarrow 0$ gives equations (41) and (42) 
variables $Y$ and $Z$. Applying the standard solution method we get the closed form solutions:

$$
\begin{gathered}
g_{y}=V(Y)=\pi(t) \frac{q(t)}{1+q(t)}, \\
g_{c}=V(Z)=\frac{\theta \frac{Z(t)}{P(t)}-\frac{Y(t)}{N}\left(\frac{W(t)}{P(t)}-2 n_{c}(t)\right)}{\theta\left(\theta+2\left(n_{c}(t)-\frac{1-r}{2}\right)\right)} .
\end{gathered}
$$

where $\pi(t)$ denotes the profits and $q(t)=(N-Y(t))^{a}(Y(t) \delta)^{b-1}$ is equivalent to the matching probability in the labor market, introduced in subsection 4.1. Equations (47) and (48) are the solutions of the intertemporal Euler equation for firms and households.

While in the zero intelligent behavior the product of $\beta$ and $g$ function vanishes, in case of rational behavior, the equilibrium dynamics depends on both the $g$ function, which embodies the rational behavior, and the uncertainty variable $\beta$. Thus, the uncertainty and the state of the macroeconomy affect the macroeconomic equilibrium and simultaneously determine the behavior of the economic agents. We can therefore identify two types of equilibria, depending on the fact that the effects of $g$ and $\beta$ offset each other or not. In the first case, since $g$ vanishes, agents' incentives do not affect the resulting equilibrium, which can be defined as uncertainty equilibrium. This equilibrium always arises in the bounded rationality setting and occurs only when $\beta \approx 0$ under the fullrationality assumption. In the second case, the product $\beta g$ does not vanish in (13): rational incentives affect the distribution of firms across states. This equilibrium corresponds to the optimal situation in which no further arbitrage would be profitable: it can be therefore qualified as rational equilibrium.

Looking at the firms' decision about production levels, equation (47) states that the macroeconomy reaches the equilibrium $\left(g_{y}=0\right)$ in two cases: null profits or, more interestingly, full employment. The latter condition is analytically represented by the no-match situation $q(t)=0$, not considering the uninteresting no-match case in which no worker supplies labor service.

For households we have a more complex result. Households behavior is in equilibrium if equation (48) is null. This leads to the macroeconomic condition:

$$
\frac{Z(t)}{P(t)}=\theta^{-1} \frac{Y(t)}{N_{z}}\left(\frac{W(t)}{P(t)}-2 n_{c}(t)\right),
$$

which can be interpreted as the demand function in terms of real wealth for each level of $n_{c}$. More precisely, the demand depends negatively on $n_{c}$ since, when $n_{c}>0$ part of the wealth in the system is not spent in consumption. When consumers are fully supply constrained $\left(n_{c}=0\right)$, the equilibrium condition becomes $\frac{Z(t)}{P(t)}=\theta^{-1} \frac{Y(t)}{N_{z}} \frac{W(t)}{P(t)}$. This condition implies the general equilibrium as all labor income $\frac{Y(t)}{N_{z}} \frac{W(t)}{P(t)}$ is consumed, there is no accumulation of wealth 
and the circular flow in the goods market is fully closed. Interestingly, if $n_{c}=$ $1 / 2$ and the real wage is equal to productivity $(W / P=1)$, the $(49)$ becomes $Z / P=0$ : if consumers are wealth-constrained and supply-constrained in the same proportion, there are no savings at the macroeconomic level.

\section{Simulations}

We run different series of simulations in order to study the dynamical system, to compare the outcomes of the agent based model and the stochastic dynamic aggregated model and to contrast the heuristic scenario with the optimizing case. $^{10}$

Simulation codes are written for Matlab and are available upon request. The parameter setting in the benchmark scenario is shown in table 3. For the stochastic dynamic aggregation, the complete simulated system is composed by equations (26) for production setting behavior, (27) for price setting behavior, (28) for wage, (29) and (30) for consumers. The system is completed by (8) and the coupled equations (9) and (10) for each of the agents' densities $n_{w}, n_{y}, n_{p}, n_{c}$.

The properties of the aggregated system are investigated in the baseline simulation presented in subsection 5.1. Then the stochastic aggregation is tested by comparing the outcomes of the aggregate model with the ABM's results in subsection 5.2, which presents the results of a Monte Carlo experiment in the form of impulse/response functions, represented as deviation from the baseline simulation. Subsection 5.3 presents the results when $g$ is optimally set.

The initial conditions for the aggregated system are set close to the macroeconomic equilibrium with the real variables (consumption, real wages and production) equal to 1 (see Table 2). The initial condition for the stochastic spread is set to 0 . Also for the ABM wages and prices are set equal to 1 and the initial worker allocation for each firm as uniform distribution given a population of 100 firms and 500 workers.

\footnotetext{
${ }^{10}$ In order to approximate the continuous time, we apply the Euler-Maruyama procedure in the interval $t \in\left(T_{0}, T_{\max }\right)$. Given the number of steps $N_{\text {step }}$ we can determine numerically the solution with the discretization of time$$
h=\frac{\left(T_{0}-T_{\max }\right)}{N_{\text {step }}} .
$$

Thus for example, we can approximate equation (20) in the following way:

$$
y(i+1)=y(i)+h\left[y(i) \delta[1+q(i)]\left(n_{y}(i)-\frac{1}{1+q(i)}\right)\right] .
$$

We generate the Wiener process with $d W=\sqrt{(h)} u$ where $u$ is distributed as a standardized Gaussian distribution.
} 


\subsection{Heuristic behavior}

Figures 1-2 show the outcome of a single simulation including in the same charts production, consumption and wealth for the aggregated system (figure 1) and the dynamics of occupation numbers for each sub-sector (figure 2).

Fluctuations and crises are due to the fact that labor and goods market incorporate search frictions at the micro-level. It is worth stressing that the macroeconomic effects of these real frictions originate from the interaction of firm and workers. Labor market frictions determine a real wage above productivity (which is constant and equal to 1 ). As the real wage is constantly above 1 , the circular flow of the system is not in equilibrium and periodic macroeconomic crisis are needed in order to re-balance the real-valued resources.

The dynamics of real variables display a quasi-stable disequilibrium in figure 1: the aggregate production level stabilizes close to the full employment equilibrium but without reaching it. Agents over-save as real average wealth is higher than 1. Moreover, real consumption always lies between supply and aggregate wealth. This is simply due to the fact that consumption is a weighted mean of supply (production) and demand (wealth). The system oscillates around the equilibrium displaying endogenous crisis, heterogeneous in duration and depth.

Three different phases can be detected in the system dynamics. The first phase (first box on the left in figure 1) can be identified by a deep recession where consumption, wealth and production display a sudden drop, causing as a consequence a fall in employment. The recession could be prolonged as the fall in employment leads to a reduction in wealth. The second phase (second box from left) is the subsequent recovery, where aggregate production, consumption and wealth grow almost to full-employment equilibrium. The real quantities steadily grow keeping consumption under production levels. This means that consumers are not supply-constrained and, at the same time, they have excess income to rebuild their stock of wealth. When quasi-full-employment equilibrium is reached, the economy enters the third phase (third box from left): wealth, consumption and production oscillate just below the full-employment equilibrium. When consumers accumulate enough wealth, aggregate demand increases, pushing firms to increase prices. Higher inflation leads to wealth devaluation followed by a fall in consumption level. The phase cannot be characterized as a recession since the fall stops when consumption and wealth hit the production level, which seems to act as a floor.

Figure 2 displays the dynamics of the occupation numbers in order to assess their evolution during the different phases. The shaded areas mark phases in which the production level is less than $95 \%$ of the full-employment equilibrium level. During these phases, a growing proportion of firms reduces production. The lower aggregate supply in goods market leads to an increase in the proportion of supply-constrained consumers $n_{c}$. Finally, due to the accumulation of inventories, more firms decrease prices causing an increase in the proportion of firms that are cost-constrained $n_{p}$. 


\subsection{Monte Carlo experiments}

In order to compare the outcomes of the ABM and the stochastic aggregation, and in particular to contrast the different responses to shocks in the parameters, we perform Monte Carlo simulations.

We follow a different approach from existing works (as for example Chiarella and Di Guilmi, 2011), comparing the results of the two solutions by testing their reaction to an exogenous shock. This approach is consistent with the fact that we run two different sets of simulations for the $\mathrm{ABM}$ and the aggregated model and, as a consequence, the results of single simulations are not comparable.

Shocks are imposed on the initial conditions defined in table 2 for: a) the aggregate production level $Y(0)(-1 \%)$; b) the aggregate price level $p(0)(+1 \%)$, c) labor productivity $(+1 \%)$ and $d)$ the interest rate $r(+1 \%)$. The plotted data are obtained by subtracting the series generated in the baseline scenario to the series with the shock in the parameters. ${ }^{11}$

In all experiments, but more clearly in the case of experiments a) and b) (figures $3-4)$, the aggregated model correctly predicts the sign of the reaction to the shock, while the magnitude of the reaction and the speed of adjustment are in some cases different. In particular, in the case of the shock in price the ABM displays a slower convergence. ${ }^{12}$

Figure 3 reports the results for experiment a). When the initial condition of employment is below the full employment level, the aggregate production and consumption also start below their equilibrium level and converge to it in the long run in both sets of simulations. When unemployment increases, workers adjust the nominal wage while the real wage remain approximately constant. This is due to the fact that firms lower their prices due to the accumulation of inventories.

Figure 4 refers to experiment b). If the initial price is above its equilibrium level, in both simulations the aggregate production drops due to a decrease in real aggregate demand. The drop in real wages leads to a larger fall in all the variables in the ABM case, which in the case of wealth prevents the adjustment to the equilibrium level within the time-frame of the simulation.

In the case of the shock to the structural parameters (technology and interest rate) the change in the $\mathrm{ABM}$ is introduced at a later stage and not at time 0 in order to appreciate the response of the system. In the case of a technology shock in experiment c) (figure 5), the ABM and the DSG-A display comparable dynamics. Production increases as expected, while real wage and wealth decreases at impact due to an increase in prices and a decrease in the number of employed workers. This result is in accordance with the New-Keynesian DSGE

\footnotetext{
${ }^{11}$ The different time scale accounts for the conversion from continuous to discrete time as specified in footnote 10 .

${ }^{12}$ The difference in the speed of convergence can be reduced by a formal calibration of the parameters aimed to a perfect match between the results of the two treatments. Given the scope and the length of the present paper, this aspect will be investigated in future research.
} 
literature, in which negative impact on employment due to technology shock is justified by price rigidities in goods or labor markets (see Liu and Phaneuf, 2013, for a survey). Aggregate consumption increases in both systems as a fraction $n_{c}$ of consumers are constrained and the increase in supply allows for a partial relaxation of market constraint.

Finally, figure 6 shows that the monetary shock has a positive impact on the economy due mainly to the implicit adaptive expectations included in this model and the fact that, in the absence of financial liabilities, monetary frictions creates positive wealth effect, leading to an increase in the aggregate available income.

\subsection{Rational optimization}

This subsection presents the results for the model introduced in section 3 and 4, using equations (47) and (48). The initial conditions are the same as in table 2 .

Figure 7 provides a detail of the simulation while figure 8 shows a larger time span. Figure 9 visualizes the dynamics of $g_{c}$ and $\beta_{c}$ while figure 10 reports the evolution of the proportion $n_{c}$ of households who are not supply constrained. Jointly examining figures $7-10$, it is evident that the system can generate two possible equilibria. Figure 7 shows that, after the phasing-in period (until around period 90), the system is in a rational equilibrium condition until around period 650. In fact, in this time-span, $g_{c}$ is equal to 0 , implying that there is no relevant difference between the payoffs of the two consumption strategies, and $b_{c} \neq 0$ (figure 9 ), which signals a low degree of uncertainty in the system. The economic interpretation is straightforward: the economic system lies in a quasistable equilibrium where uncertainty is low and consumers' utility and firms' profits are maximized. Thus, when the system is in the rational-equilibrium, the $\beta_{c}$ variable, following the macro-variables' dynamics, is far from zero as expected. The rational equilibrium is associated with two emergent facts: employment is full as the production reaches the maximum level, with employment equal to $N_{z}=500$ and 1 unit of consumption per capita, and wealth lies near production (figure 7). However, the endowment of real wealth is higher than unity due to goods market frictions, allowing for a level of consumption higher than aggregate production. As for the households behavior, $n_{c}$ (the proportion of wealth-constrained households) fluctuates around 0.5 during the same period (figure 10). The stability of this quantity (net of the stochastic noise) is due to the fact that there are no incentives for the agents to change strategy.

Since we introduce rationality only in production and consumption decisions, prices and wages are still set in a sub-optimal fashion. This setting, together with the interaction in the goods and labor market, allows for real inefficiencies: real salary is always higher than productivity. This affects the long run behavior of the economy but in a different manner with respect to the heuristic case. Figures 7 and 8 show that, around period 650, the rational equilibrium no longer holds due to dynamic inefficiencies in the labor and goods market. Instead of 
periodic crisis, the system is caught in what we can identify as an uncertainty trap (Aoki and Yoshikawa, 2006): a scenario in which increasing uncertainty locks the macroeconomy away from the optimal equilibrium.

As shown by figures 7 and 8, households reduce consumption and increase savings. This creates a growing distance between wealth and the other variables. Soon after period 650 , wealth rapidly increases, diverging from the rational equilibrium level. As the dynamics of $g_{c}$ and $\beta_{c}$ demonstrates, the system transitions to the uncertainty equilibrium in which economic behavior is not rational $\left(g_{c} \neq 0\right)$, uncertainty is high, as shown by $\beta_{c} \rightarrow 0$ (figure 9) and consumers become fully supply-rationed $\left(n_{c} \rightarrow 0\right)$. The inefficiencies determine a growing uncertainty, as $\beta_{c}$ approaches 0 until period 650 , when it hits a peak since a large fraction of consumers switch to the supply-constrained state. This determines a discontinuity in $g_{c}$ (capped at 100 in the simulations), which subsequently takes large negative values due to the negative denominator when $n_{c} \rightarrow 0$ in equation (48). From around period $700, \beta_{c}$ remains at 0 signaling high uncertainty.

With reference to equation (37), the simulations demonstrate that the effects of the (dis)equilibrium in the goods market dominates the effects of the agents' choices. In fact, in the rational equilibrium $\beta \neq 0$ even when $n_{c} \approx 1 / 2$ because $\frac{P(t) Y(t)}{N_{z}(t)} \approx Z(t)$, while we have uncertainty equilibrium even with $n_{c}=0$ due to the disequilibrium in the goods market. These numerical results also integrate the analysis of equation (49), showing that, even when $g=0$, the inefficiencies that are still present can lead to a shift in the pattern of evolution of the economy. The analysis of section 6 below provides further insights on this point.

The results of this section can be summarized by the two following propositions:

Proposition 1. When agents behave as atoms, the real inefficiency affecting the system is managed by the economy through periodic crises: agents recover aggregate losses diminishing production, income and aggregate demand then returning to the equilibrium level.

Proposition 2. When rational agents live in a complex interacting world with real or nominal inefficiencies the economy can experience the uncertainty trap in the long run.

In the heuristic treatment, since the behavior of agents is stochastic, small idiosyncratic shocks can trigger feedback effects at the agent-level that originate the cyclical crises. In the optimization case, the accumulation of wealth is selfsustaining, as shown by equation (48): the perceived relative pay-offs of the consumption behaviors change and, in the absence of re-equilibrating mechanisms for the nominal variables, this leads to a permanent difference between wealth and consumption. 


\section{Analytical identification of the equilibria}

As a final step, we identify the critical levels of the proportion of supplyconstrained consumers that determine the transition from one equilibrium to another. The visual inspection of figure 10 can be integrated by the analysis of the potential, introduced in section 2. Besides identifying the critical points, this study defines the possible transition paths between different attractors and the conditions in which our artificial economy can be locked in the uncertainty trap. We focus on the households subsystem since it allows us to describe the evolution of wealth and consumption demand.

\subsection{Identification of the equilibrium type and transitions}

The potential function (14) for the number of not-rationed consumers is

$$
U=-2 \int_{0}^{n_{c}} g_{c}(t) d Z-\frac{H\left(n_{c}, t\right)}{\beta\left(n_{c}, t\right)},
$$

with $g_{c}$ defined by (48). The critical points of (52) are the equilibria for the occupation number $n_{c}$ and therefore for aggregate wealth.

Considering equation (2) and (52), the first order condition is

$$
U^{\prime}=-2 g_{c}(t)-\frac{H^{\prime}\left(n_{c}(t)\right)}{\beta\left(n_{c}(t)\right)}+\frac{\beta^{\prime}\left(n_{c}(t)\right)}{\beta\left(n_{c}(t)\right)^{2}}=0,
$$

With respect to the formula (16) presented in subsection 2.1, equation (53) introduces the term $\frac{\beta^{\prime}(n c)}{\beta(n c)^{2}}$. This term is not present in the heuristic treatment and represents the additional knowledge available to the social planner, which is not usable by the boundedly rational agents.

Since a closed form solution cannot be derived analytically, we evaluate it numerically. Figures 11-13 display the results of the numerical analysis. Due to the discontinuity around $n_{c}=0.001$, in order to make critical points and discontinuities visible we need to split the plot in tree sub-regions: one near $n_{c}=0$ and two around $n_{c}=0.5$. Figure 11 plots the whole function with the zoom on the areas around the critical point for an overall assessment. Figure 12 provides a better details of the critical points. The potential has two local minima at $n_{c, 1}=0$ and $n_{c, 2}=0.48$ and the two points are separated by a discontinuity. Consequently, the transition between the two equilibria is not possible. The first panel of figure 12 shows also a local maximum that splits the region on the left. In the local minimum $n_{c, 1}=0$, from the simulations we can verify that $\beta_{c}=0$ and $g_{c} \neq 0$ (figures 9 and 10): the uncertainty is maximal and the equilibrium is not rational. The middle panel of figure 12 shows the neighborhood of $n_{c, 2}=0.48$, that is associated with $g\left(n_{c}\right)=0$ (figures 9 and 10) and can be therefore identified as a rational equilibrium. Also, since $\lim _{n_{c} \rightarrow n_{c, 2}} \beta\left(n_{c}\right)=-\infty$, as the economy approaches the socially optimal point, 
uncertainty reduces. In the absence of nominal frictions the rational equilibrium is therefore stable. The macroeconomic signal that agents receive drives them not to change their behavior. Finally, we have a third extreme point locate in $n_{c}=0.51$ that is a maximum. The critical points are clearly visible in figure 13 where the $\log \left(U^{\prime}\right)$ is displayed.

\subsection{Policy Implications}

The model presented above is parsimonious and serves the purpose of illustrating the usefulness of the analytical tools. Nevertheless, it is possible to run a simple policy experiment to assess how a policy maker can influence the type of equilibrium that the system can achieve. Within the present framework, the task of the policy maker is to prevent the system from being locked in the uncertainty equilibrium or at least to reduce the likelihood of its occurrence. In fact, in the uncertainty equilibrium, agents may have contrasting incentives and this coordination failure can jeopardize the success of the policy measures. The following analysis focuses on the impact of interest rate changes on the equilibrium condition in the household sector, being the interest rate the only policy variable in the model.

The goal is to investigate the effect of a zero lower bound on the number and the characteristics of the equilibria and to assess whether the rational and uncertainty equilibria are affected by the level of the interest rate.

We run three different simulations of the potential function for $r=[-0.005,0,0.005]$. The equilibrium values for per-capita production and real wage are equal to 1 and 1.005, respectively, as obtained in the simulations, while $\delta=0.005$. Figure 14 replicates figure 13 for the different values of the interest rate, which is set to 0.001 in the baseline scenario. Clearly, the number and the type of equilibria change as $r$ moves from positive to negative values. In case of a positive interest rate the results do not substantially differ from the baseline simulations: the two possible outcomes are rational and the uncertainty equilibrium. For $r=-0.005$ the system can avoid the uncertainty equilibrium and the only attractors are the two rational equilibria in the neighborhood of $n_{c}=0.5$. Also the discontinuity near the uncertainty equilibrium disappears, so the system can adjust to the rational equilibrium in the long run. This is due to the fact that the negative interest rate prevents the accumulation of wealth in the long period. The uncertainty equilibria disappears also in the case of zero interest rate but indeterminacy arises since no critical point can be identified.

To conclude, the zero lower bound can provide a source of indeterminacy and can lead the economy into the uncertainty trap. If real factors of instability originating from the interaction of the agents are not eliminated, negative nominal interest rates may be needed in order to curb the effects of uncertainty. While this result is well known in macroeconomic theory, the DSG-A approach can detect the conditions under such a situation occurs and determine the structure of equilibria through the analysis of the potential. 


\section{Conclusions}

This paper tests the Dynamic Stochastic Generalized Aggregation approach (DSG-A) as a methodology to map complexity arising from explicit agent interaction into different levels of rationality in agents' behavior. We apply the DSG-A approach to a medium-scale ABM, providing two main contributions. First, we estimate a set of macro equations starting from the micro behaviors of different sets of agents (namely firms, workers and consumers). Building from simple micro-behavioral rules, we elaborate a macro-model that is able to qualitatively replicate the results produced of the ABM. This kind of methodology can be helpful in analytically evaluating the properties of complex models obtaining reduced forms in order to validate and estimate ABMs.

The second contribution is in that, by assuming that the proportions of agents adopting one or the other strategy are optimally set, it is possible to isolate the effects of rational incentives and uncertainty. In particular, the numerical and analytical treatments identify two types of equilibria: the rational equilibrium where all the opportunities for welfare increase are exhausted, and the uncertainty equilibrium, which is the result of the system complexity.

To summarize the results, in presence of market inefficiencies, the accumulation of wealth determines over-saving and over-consumption leading to periodic crises in the heuristic setting and to the uncertainty trap in the optimizing setting. The analytical investigation and the simulations show that, in the optimizing case for consumption, the system has two attractors, which are associated to, respectively, an uncertainty equilibrium and a rational equilibrium. The analysis of the potential function is also applied in a simple policy exercise. In a world where agents are rational but interact in a complex economy multiple equilibria are possible and monetary policy can affect their number and quality. In particular, the zero lower bound limits the capacity of the policy maker to avoid the uncertainty trap.

This promising methodology can be employed to build more general analytical models including in principle the possibility to include standard structural macroeconomic models (DSGE) as special cases. The full exploration of this possibility is the next item of our research agenda. A comparison with DSGE models can also involve the treatment of the transition rates. As mentioned in the paper, the method presented here endogenously generates the transition rates and could be used for a comparison with the empirically estimated rates presented in DSGE with Markov switching. The approach can be also extended by estimating the actual probability of the uncertainty trap in a real economy by using a model calibrated with empirical data. 


\section{A Entropy maximization}

This appendix presents the solution of the system (13). The goal is to maximize the Entropy:

$$
H=-n_{1} \log \left(n_{1}\right)-n_{2} \log \left(n_{2}\right)
$$

subject to the constraints

$$
\left\{\begin{array}{l}
n_{1}+n_{2}=1, \\
V_{1} n_{1}+V_{2} n_{2}=\dot{Y} .
\end{array}\right.
$$

The first constraint is a simple normalization constraint, while the second can be considered as an accounting identity. For example, if $V_{1}$ and $V_{2}$ are the change in production for the firms of, respectively, type 1 and type 2 , the variation in total output must be equal to $\dot{y}$. The Lagrangian for this problem is

$$
-n_{1} \log \left(n_{1}\right)-n_{2} \log \left(n_{2}\right)+\delta_{1}\left(n_{1}+n_{2}-1\right)+\delta_{2}\left(V_{1} n_{1}+V_{2} n_{2}-\dot{Y}\right)
$$

The first order conditions are given by

$$
\begin{aligned}
& \frac{d L}{d n_{1}} \Rightarrow-\log \left(n_{1}\right)-1+\delta_{1}+\delta_{2} V_{1}=0, \\
& \frac{d L}{d n_{2}} \Rightarrow-\log \left(n_{2}\right)-1+\delta_{1}+\delta_{2} f_{2}=0, \\
& \frac{d L}{d \delta_{1}} \Rightarrow n_{1}+n_{2}=1, \\
& \frac{d L}{d \delta_{2}} \Rightarrow \dot{Y}=V_{1} n_{1}+V_{2} n_{2} .
\end{aligned}
$$

Imposing the following identities

$$
\begin{aligned}
& \delta_{1}=1-\alpha \\
& \delta_{2}=-\beta,
\end{aligned}
$$

the first two equations in (A.7) become

$$
\begin{aligned}
& n_{1}=e^{-\left(\alpha+\beta V_{1}\right)}, \\
& n_{2}=e^{-\left(\alpha+\beta V_{2}\right)} .
\end{aligned}
$$

Substituting the above equations into the third in (A.7) we obtain

$$
e^{-\alpha}=\frac{1}{e^{-\beta V_{1}}+e^{-\beta V_{2}}},
$$

which, substituted in the last of (A.7) gives

$$
\left(V_{1}-\dot{Y}\right) e^{-\beta V_{1}}+\left(V_{2}-\dot{Y}\right) e^{-\beta V_{2}}=0
$$


Rearranging we obtain an expression for $\beta$

$$
\beta=\left(V_{2}-V_{1}\right)^{-1} \log \left(\frac{n_{1}}{1-n_{1}}\right),
$$

Since

$$
\begin{aligned}
& n_{1}=e^{-\alpha(t)} e^{-\beta(t) V_{1}(t)}, \\
& n_{2}=e^{-\alpha(t)} e^{-\beta(t) V_{2}(t)} .
\end{aligned}
$$

Then

$$
\begin{aligned}
& p_{1}=\frac{e^{-\beta(t) V_{1}(t)}}{Z}, \\
& p_{2}=\frac{e^{-\beta(t) V_{2}(t)}}{Z},
\end{aligned}
$$

where $Z$ represents the partition function:

$$
Z=e^{-V_{1}(t) \beta(t)}+e^{-V_{2}(t) \beta(t)} .
$$

\section{B Derivation of the aggregate variables}

\section{B.1 Production}

The firms are the micro units and production is the state quantity. Firms can choose two different strategies, and, therefore, can be in two possible different states, depending on the value of $S_{i}$, as defined by (20). For firms in the first state, the quantity is adjusted up if the stock $S$ in the previous period is empty: $S_{i}(t-d t)=0$. Otherwise, the quantity is set down if there was a supply excess: $S_{i}(t-d t)>0$. The stock $S$ is a signal of the fitness of the strategy to set the quantity.

\section{B.1.1 Dynamics}

Firms are in state 0 if they revise their production upward and in state 1 otherwise. The aggregate excess supply ratio is

$$
w_{z}(t)=\frac{Y(t) P(t)}{N_{z} C(t)},
$$

where $P(t)$ is the aggregated price index, $C(t)$ the aggregated nominal demand, $N^{z}$ is the expected number of visits and $Y(t)$ is the aggregated supply. In the aggregate this index must be equal to one. We assume that, due to the matching 
frictions, for each single consumer $w_{z, j} \in[0.75,1.25]$. Let us define the stock variable as the excess demand

$$
S_{i}(t)=Y_{i}(t)-\sum_{z=1}^{N_{z}} w_{z}(t)
$$

We assume that each consumer is willing to spend all her endowment and that the matching process in the market implies that probability distribution of $w_{z}$ across consumers is uniform.

The equation governing the aggregated variable is the weighted mean of the variation in production for firms that reduce production and firms that are able to increase production, depending on the matching the labor market. Using (25) the aggregate equation can be written as

$$
\dot{Y}(t)=Y(t) \delta(1+q(t))\left(n_{y}(t)-\frac{1}{1+q(t)}\right) .
$$

where $q(t)=u_{n}(t)^{a}(Y(t) \delta)^{b-1}$ is the matching probability in the labor market for the unemployed worker.

\section{B.1.2 Transition probabilities}

Imposing equation (B.2) to be equal to zero, we can define the level of production in correspondence to the threshold level of null excess demand as $w_{z}=\frac{Y_{i}(t)}{N^{z}}$. Then, using the known cumulative probability of $w_{z}$, we can define the probability to entry and exit to/from the state 1 respectively as

$$
\zeta_{y}(t)=F\left(\bar{w}_{z}(t)\right)=p\left(\tilde{w}_{z} \leq \bar{w}_{z \zeta}(t)\right)=\frac{\bar{w}_{z}(t)-0.75}{0.5},
$$

re

$$
\iota_{y}(t)=1-F\left(\bar{w}_{z}(t)\right)=p\left(\tilde{w}_{z} \leq \bar{w}_{z \iota}(t)\right)=1-\frac{\bar{w}_{z}(t)-0.75}{0.5} .
$$

In case of production, the payoffs for the alternatives $V_{1}$ and $V_{2}$ used to derive $\beta$, according to (2) and $g$ for the heuristic treatment, according to (17), are, respectively, $V_{1}=u^{a}(Y \delta)^{b}$ in case of the increasing strategy and $V_{2}=-\delta Y$ for the decreasing strategy. The payoff $V_{1}$ is simply represented by the matching function since, given (21) and (25), the increase in production is bounded upward by the number of workers that the firm can recruit on the job market.

\section{B.2 Prices}

Firms revise their price up (down) if $S=0(S>0)$. 


\section{B.2.1 Dynamics}

The law of motion of price is

$$
\Delta p_{i}(t)=p_{i}(t-d t) \times\left\{\begin{array}{l}
\delta \text { if } S_{i}(t)=0 \\
-\delta \text { if } S_{i}(t)>0
\end{array} .\right.
$$

A firm will actually apply the variation calculated as in (B.6) only if it is able to cover at least the production costs. If we indicate with $p^{1}$ this minimum threshold we can write

$$
p_{i}^{1}(t)=\frac{\sum_{k \in h_{l}} w_{k}(t)}{y_{i}(t)}
$$

i.e. the numerator is the firm's wage bill. So the actual price for the firm $i$ at time $t$ will be equal to

$$
p_{i}(t)=\left\{\begin{array}{l}
p(t-d t)+\Delta(p)_{i}(t) \text { if } p(t-d t)+\Delta(p)_{i}(t) \geq p_{i}^{1}(t) \\
p_{i}^{1}(t) \text { if } p(t-d t)+\Delta(p)_{i}(t)<p_{i}^{1}(t)
\end{array} .\right.
$$

Considering that the sign of the price variation is the same of the variation in the production, we can approximate (B.6) as

$$
\dot{p}_{i}(t)=\left\{\begin{array}{l}
p_{i}(t) \delta \\
-p_{i}(t) \delta
\end{array}\right\}=\operatorname{sign}\left(\dot{y}_{i}\right) p_{i}(t) \delta .
$$

Subtracting from both size of the (B.7) the expression $-\left(w^{s}(t)-1+p_{i}(t-d t)\right)$ and rearranging we obtain

$$
p_{i}(t)-p_{i}(t-d t)-w_{i}^{s}(t-d t)=w_{i}^{s}(t)-w_{i}^{s}(t-d t)-p_{i}(t-d t)
$$

Approximating and rearranging again it is possible to write

$$
\dot{p}(t)=\dot{w}^{s}(t)+w^{s}(t)-p(t) .
$$

A fraction $n_{p}$ of firms set the price according to the rule (B.6); the remaining firms are cost constrained and adopt the price $p_{i}^{1}$. Thus, the average change in price will be equal to

$$
\dot{p}(t)=n_{p}(t)(\operatorname{sign}(\dot{y}) p \delta)+\left(1-n_{p}(t)\right)\left(\dot{w}^{s}(t)+w^{s}(t)-p(t)\right),
$$

which can be rewritten as

$$
\dot{p}(t)=n_{p}(t)\left(\operatorname{sign}(\dot{y}) p(t) \delta+p(t)-w^{s}(t)-\dot{w}^{s}(t)\right)+\dot{w}^{s}(t)+w^{s}(t)-p(t) .
$$

\section{B.2.2 Transition probabilities}

We can define a random variable as $v_{p}=P(t)-W(t)$ as the difference between the aggregate price index and satisficing wage. We assume that $v_{p} \sim N(0,1)$ and the transition probabilities are

$$
\zeta_{p}(t)=F\left(v_{p}(t)<\bar{v}_{p}\right)=\frac{1}{2}\left[1+\operatorname{erf}\left(\frac{v_{p}(t)}{\sqrt{2}}\right)\right],
$$




$$
\iota_{p}(t)=1-\zeta_{p}(t) .
$$

Considering (24), the payoffs for the two conditions of price increasing and the cost-constrained price are, respectively $V_{1}=\operatorname{sign}(\dot{Y}) P \delta$ and $V_{2}=\dot{W}+W-P$.

\section{B.3 Workers}

Workers rise (reduce) their satisficing wage if they were employed (unemployed) in the previous unit of time.

\section{B.3.1 Dynamics}

Workers set their satisficing wages $w^{s}{ }_{j}$ given their occupational status:

$$
\dot{w}_{j}^{s}(t)=\begin{array}{ll}
w_{j}^{s}(t) \delta & \text { if } o c c_{j}(t)=1 \\
-w_{j}^{s}(t) \delta & \text { if } o c c_{j}(t)=0
\end{array} .
$$

We follow Russo et al. (2007) and assume workers to have a complete market power because they are able to extract all the surplus in the labor market bargaining. Indeed, a worker earns his satisficing wage if hired, otherwise he stays unemployed. At the aggregate level, (B.14) can be written as

$$
\dot{W}^{s}(t)=W^{s}(t) \delta\left(n_{w}(t)-\frac{1}{2}\right) .
$$

\section{B.3.2 Transition probabilities}

At each point in time, a single worker can occupy one of the two states: "employed" $(o c c(t)=1)$ or "unemployed" $(o c c(t)=0)$. We are interested in specifying the jump-process transition probabilities of the worker for the state "employed". The hiring procedure is modeled as a random draw from a double urn. The first draw decides whether a worker is employed or unemployed and the second if the firm adjusts production quantity up or down. The possible combined events are four: a) if the firm adjusts upward and the worker is employed the probability is $1 ; \mathrm{b}$ ) if the firm adjusts downward and worker is employed there is a positive probability to be fired; c) if the firm adjusts upward and the worker is unemployed there is a positive probability to be hired; d) if the firm adjusts downward and the worker is unemployed the probability is zero. To sum up, the different four events are:

$$
\begin{aligned}
& M_{1}=Y^{+} \cap o c c(t-d t)=1 \\
& M_{2}=Y^{-} \cap \operatorname{occ}(t-d t)=1 \\
& M_{3}=Y^{+} \cap \operatorname{occ}(t-d t)=0 \\
& M_{4}=Y^{-} \cap \operatorname{occ}(t-d t)=0
\end{aligned} .
$$


We define $P(o c c(t)=1)$ as the probability to jump in the employed state. This probability can be quantified as the conditioned probability over the four distinct events defined by (B.16):

$$
P\left(\text { occ }_{t}=1\right)=\sum_{k=1}^{4} P\left(\text { occ }_{t}=1 \mid M_{k}\right) P\left(F_{k}\right) .
$$

Defining $w^{s}$ as an arbitrary satisficing wage level, all the above considerations can be formally expressed in the following way

$$
\begin{aligned}
& P\left(\operatorname{occ}(t) \mid M_{1}\right)=1 \\
& P\left(\operatorname{occ}(t) \mid M_{2}\right)=1-M\left(w<w^{s}\right) \\
& P\left(\operatorname{occ}(t) \mid M_{3}\right)=M\left(w<w^{s}\right) \\
& P\left(\operatorname{occ}(t) \mid M_{4}\right)=0
\end{aligned}
$$

Substituting these expressions in equation (B.17) we have

$$
\begin{aligned}
P(\text { occ }(t)=1)= & P\left(Y^{+}\right) P(\operatorname{occ}(t)=1)+\left(1-M\left(w<w^{v}\right)\right) P\left(Y^{-}\right) P(\text { occ }(t)=1)+ \\
& +M\left(w<w^{v}\right) P\left(Y^{+}\right) P(\text { occ }(t)=0),
\end{aligned}
$$

Or

$$
P(\operatorname{occ}(t)=1)=P(\operatorname{occ}(t)=1)\left[1-M\left(w<w^{v}\right)\right]+M\left(w<w^{v}\right) P\left(Y^{+}\right) .
$$

In this case we have a dynamic equation for the transition probabilities. We need to specify the probability to be hired or fired. Firms collect workers satisficing wage and sort them in ascending order. We assume that information about wage distribution $F(w)$ over the whole workers population is known. This means that if in aggregate the economy opens a positive number of vacancies $(d y / y>0)$ the aggregate system is evaluating the left tail distribution. Otherwise, if the system is destroying jobs $(d y / y<0)$, the evaluation is on the right tail. The firms sort the applications in the ascending order of the reservation wage. Let $v$ be the number of vacancies opened in the system at a given time; hence the probability for a worker of being hired is equal to the probability of having a reservation wage lower than the lowest $v$ wages in the system. Analogously, if a worker is employed in a firm that is reducing its labor force, his probability of loosing the job is equal to the probability of having a reservation wage higher of a certain threshold. We can assume that the right tail is populated by workers with a long period of continuous employment, while the left tail represents those workers coming from a relatively long period of unemployment and therefore forced to demand a low wage in order to be hired.

We assume a standardized normal distribution for the variable $x=-\alpha /(\dot{Y} / Y 100)$. The hyperbolic tangent provide us a behavior such as $x \rightarrow+\infty$ if $\dot{Y} / Y \rightarrow 0^{+}$ 
and $x \rightarrow-\infty$ if $\dot{Y} / Y \rightarrow 0^{-}$. Finally we can evaluate the cumulative probability as $F=1 / 2-1 / 2 \operatorname{erf}(x / \sqrt{(} 2))$.

If we consider the equation in continuous time we have

$$
\dot{P}(\operatorname{occ}(t)=1)=(\eta-P(\operatorname{occ}(t)=1)) F .
$$

According to (22), the payoffs associated to the salary-increasing and salarydecreasing strategies are, respectively, $V_{1}=W \delta$ and $V_{2}=-W \delta$.

\section{B.4 Consumers}

For consumers in state 1 demand is set simply as the real wealth; for consumers in state 2 , demand is equal to the supplier firm's output because constrained.

\section{B.4.1 Dynamics}

We set $n_{c}$ as the occupation number of the state 1 and aggregate equation is:

$$
C(t)=n_{c}(t) Z(t)+\left(1-n_{c}(t)\right) \frac{P(t) Y(t)}{N_{z}(t)},
$$

where $Z$ is the wealth. Equation (B.22) states that aggregate consumption is exactly the weighted mean between aggregate wealth and aggregate supply. If $n_{c}$ goes to zero we have a full constrained market.

Defining the number of jobless workers as $1-\frac{Y(t)}{N_{z}(t)}$, total wealth evolves according to:

$$
\dot{Z}(t)=\left[r Z(t)+W^{s}(t)-C(t)\right] \frac{Y(t)}{N_{z}(t)}+[r Z(t)-C(t)]\left(1-\frac{Y(t)}{N_{z}(t)}\right) .
$$

\section{B.4.2 Transition probabilities}

Let us define the variable $v_{c}=\frac{P(t) Y(t)}{Z(t) N_{z}}$. We assume that $v_{c} \sim N(0,1)$ and the transition rates are

$$
\begin{gathered}
\zeta_{c}(t)=F\left(v_{c}(t)<\bar{v}_{c}\right)=\frac{1}{2}\left[1+\operatorname{erf}\left(\frac{v_{c}(t)}{\sqrt{2}}\right)\right], \\
\iota_{c}(t)=1-\zeta_{c}(t) .
\end{gathered}
$$

For consumers who are not supply-constrained the payoff is simply given by the consumption $V_{1}=\frac{P Y}{N}$, while for those who accumulate involuntary savings the payoff is given by the wealth: $V_{2}=Z$. 


\section{Derivation of macroeconomic equations}

Taking production as an example and assuming away the matching constraint in (B.3), consider the discrete time rule

$$
y_{i}(t)=y_{i}(t-1) \times\left\{\begin{array}{l}
(1+\delta) \text { if } S_{i}(t-1)=0 \\
(1-\delta) \text { if } S_{i}(t-1)>0
\end{array} .\right.
$$

Subtracting $y_{i}(t-1)$ the left and right sides from the equal sign, dividing by $d t$ and assuming $d t \rightarrow \infty$, we get

$$
\dot{y}_{i}(t)=y(t) \times\left\{\begin{array}{l}
+\delta \text { if } S_{i}(t-1)=0 \\
-\delta \text { if } S_{i}(t-1)>0
\end{array} .\right.
$$

Then in order to get the macro rule for $Y(t)$ we need to know time evolution for $n_{y}(t)$ to get

$$
\dot{Y}(t)=Y(t)\left[n_{y}(t) \delta-\left(1-n_{y}\right) \delta\right]=2 \delta Y(t)\left(n_{y}(t)-\frac{1}{2}\right)
$$




\section{Tables and figures}

\begin{tabular}{lcll}
\hline Agent & States & Fraction & Description \\
\hline \multirow{2}{*}{ Firm's price } & $p^{1}$ & $n_{p}$ & Free adjustment \\
& $p^{0}$ & $1-n_{p}$ & Unitary cost constrained \\
\hline \multirow{2}{*}{ Firm's quantity } & $y^{+}$ & $n_{y}$ & Upward adjustment \\
& $y^{-}$ & $1-n_{y}$ & Downward adjustment \\
\hline \multirow{2}{*}{ Workers wage $w^{s}$} & $o c c^{1}$ & $n_{w}$ & Upward adjustment \\
& $o c c^{0}$ & $1-n_{w}$ & Downward adjustment \\
\multirow{2}{*}{ Workers' consumption } & $c^{1}$ & $n_{c}$ & Not rationed \\
& $c^{0}$ & $1-n_{c}$ & Rationed \\
\hline
\end{tabular}

Table 1: Agents and States

\begin{tabular}{ll}
\hline Symbol and initial value & Description \\
\hline$y=480$ & Aggregate Production \\
$p=1$ & Price \\
$w=1$ & Wage \\
$c=1$ & Consumption \\
$z=1$ & Wealth \\
$m_{y}=0.5$ & Drift for production \\
$s_{y}=0$ & Spread for production \\
$m_{p}=0.5$ & Drift for price \\
$s_{p}=0$ & Spread for price \\
$m_{w}=0.5$ & Drift for wage \\
$s_{w}=0.1$ & Spread for wage \\
$m_{c}=0.01$ & Drift for supply constrained consumers \\
$s_{c}=0$ & Spread for supply constrained consumers \\
\hline
\end{tabular}

Table 2: Initial conditions. 


\begin{tabular}{ll}
\hline Symbol and value & Description \\
\hline$\delta=0.01$, & Price and wage adjustment rate \\
$r=0.1 \%$, & Interest rate \\
$a=0.5$, & Matching function parameter \\
$b=0.5$, & Matching function parameter \\
$h_{w}=h_{y}=3$ & Subset of firms for each workers/consumers in labor \\
& and goods market \\
$k=0$ & Searching cost in goods market \\
$N=500$ & Consumers \\
\hline
\end{tabular}

Table 3: Parameters

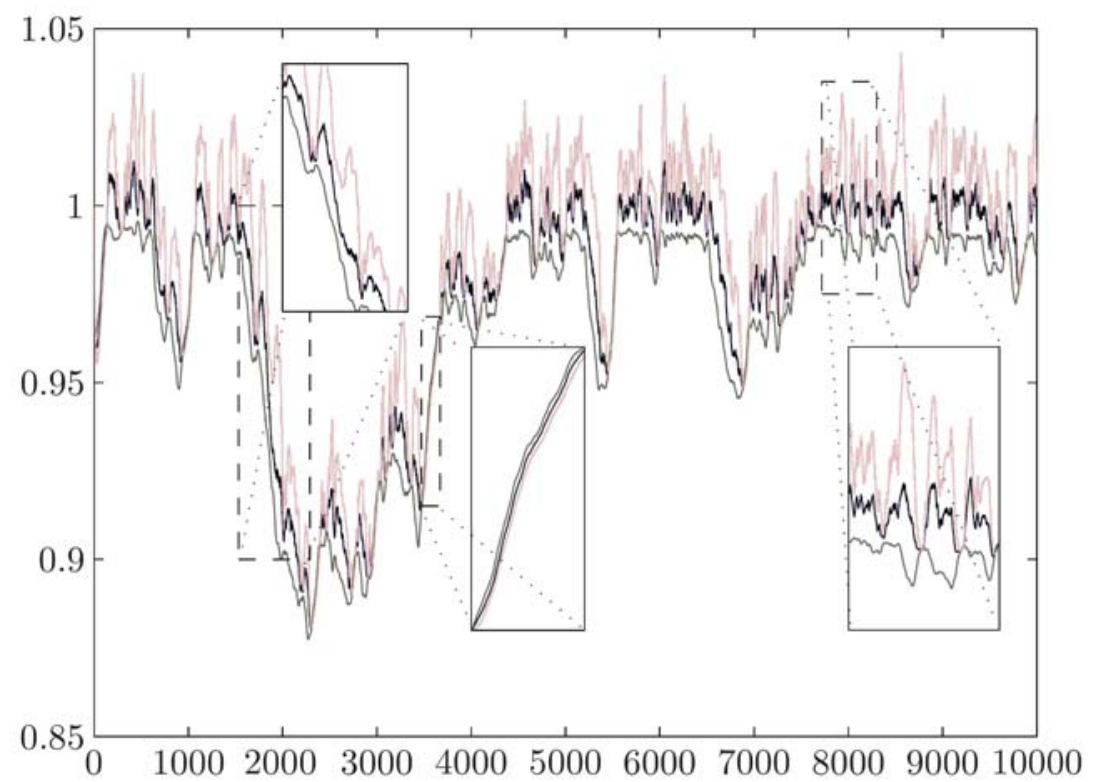

Figure 1: Simulation of the system for the aggregated model. Black line: percapita production, light gray line: per-capita wealth, dark gray line: per-capita consumption.

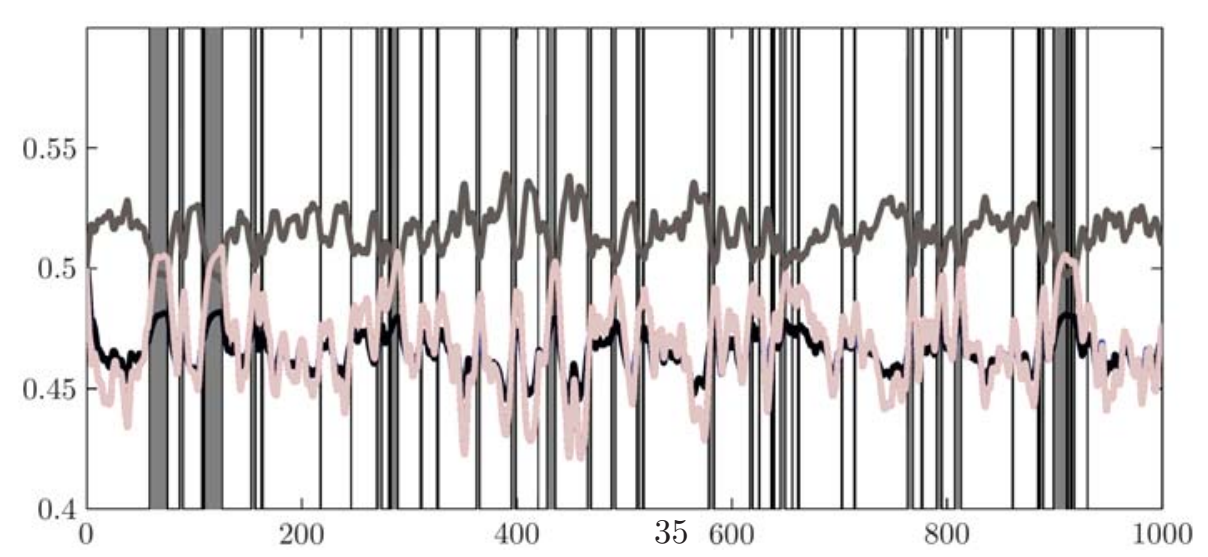

Figure 2: Simulation of the system for the aggregated model: occupation numbers. Light gray line: increasing-production firms $\left(n_{y}\right)$, dark gray line: notrationed consumers $\left(n_{c}\right)$, black line: not cost-constrained firms $\left(n_{p}\right)$. Shaded areas: periods in which the production level is less than $95 \%$ of the fullemployment equilibrium level. 

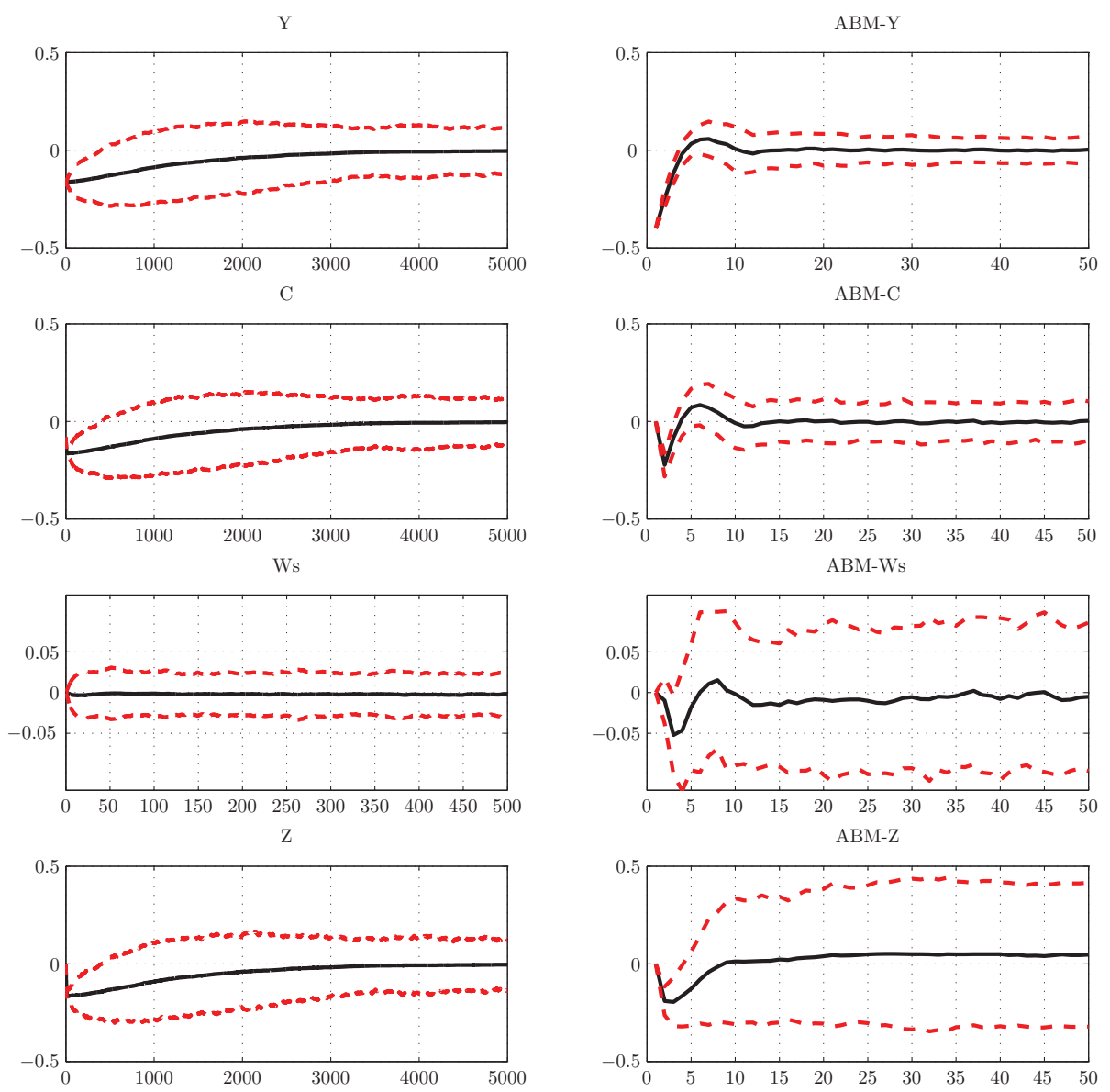

Figure 3: Shock in Production (-1\%). Monte Carlo simulation with 1000 replications. Percentage deviation from baseline simulation and confidence intervals. Left panels: DSG-A system; right panels: ABM. 

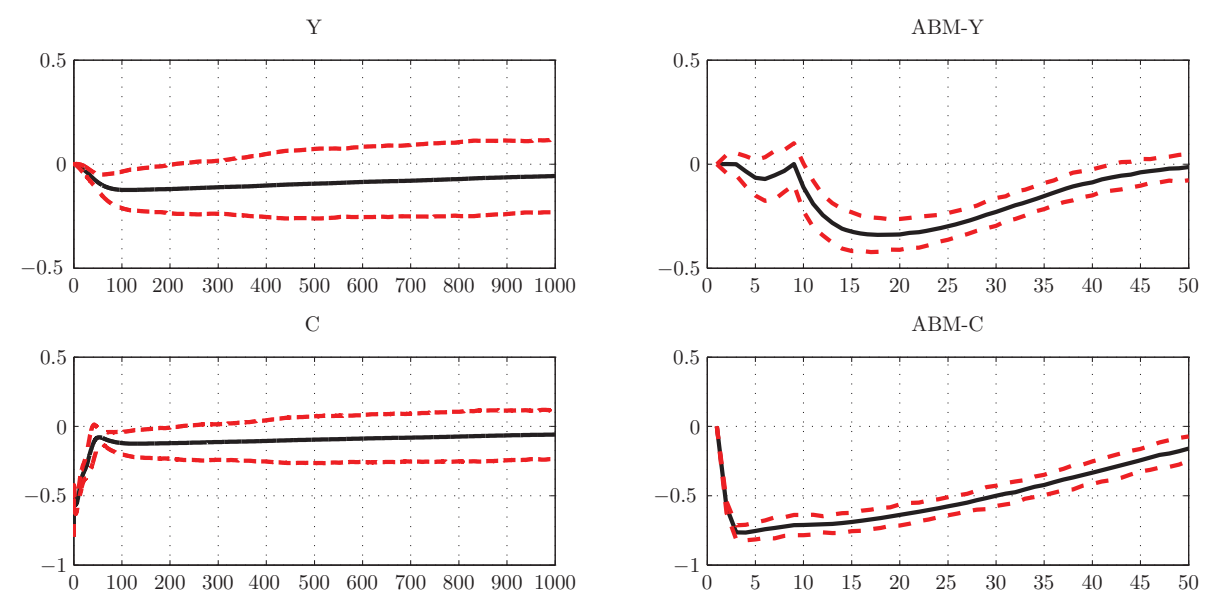

Ws
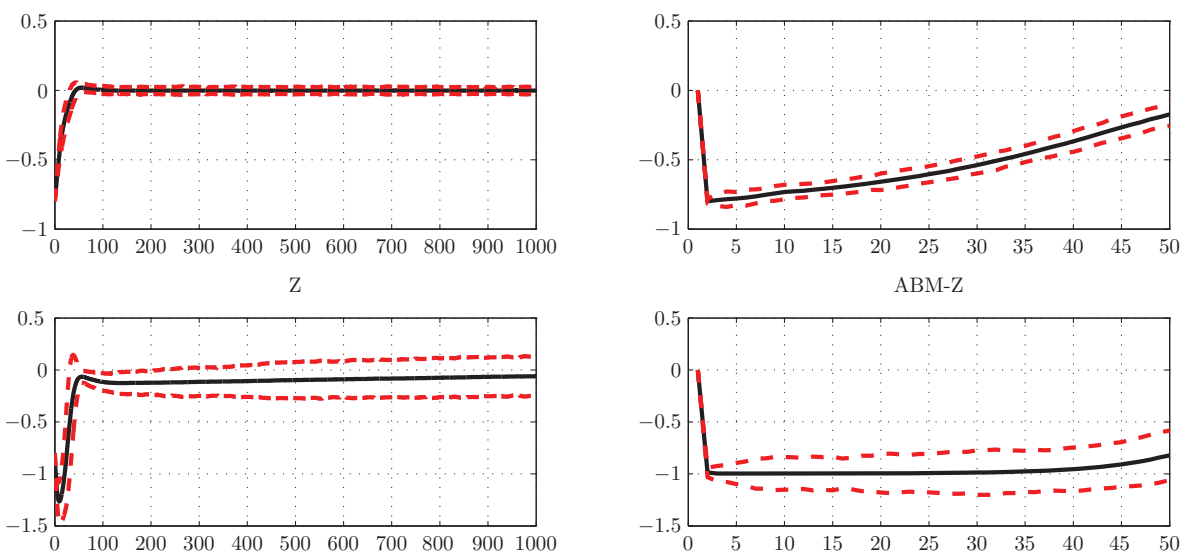

Figure 4: Shock in Prices (+1\%). Monte Carlo simulation with 1000 replications. Percentage deviation from baseline simulation and confidence intervals. Left panels: DSG-A system; right panels: ABM. 

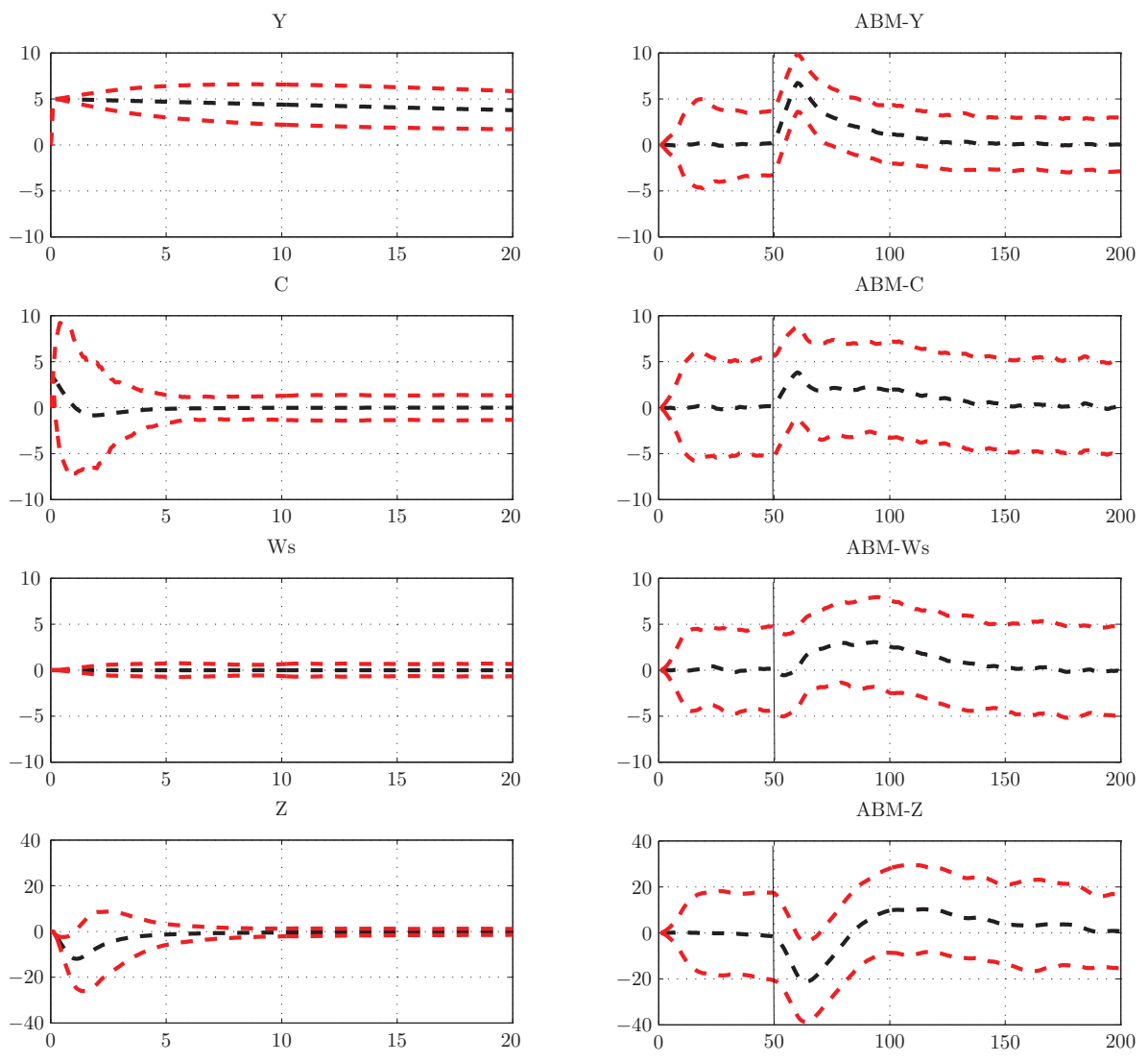

Figure 5: Shock in labor productivity $(+1 \%)$. Monte Carlo simulation with 1000 replications. Percentage deviation from baseline simulation and confidence intervals. Left panels: DSG-A system; right panels: ABM. 

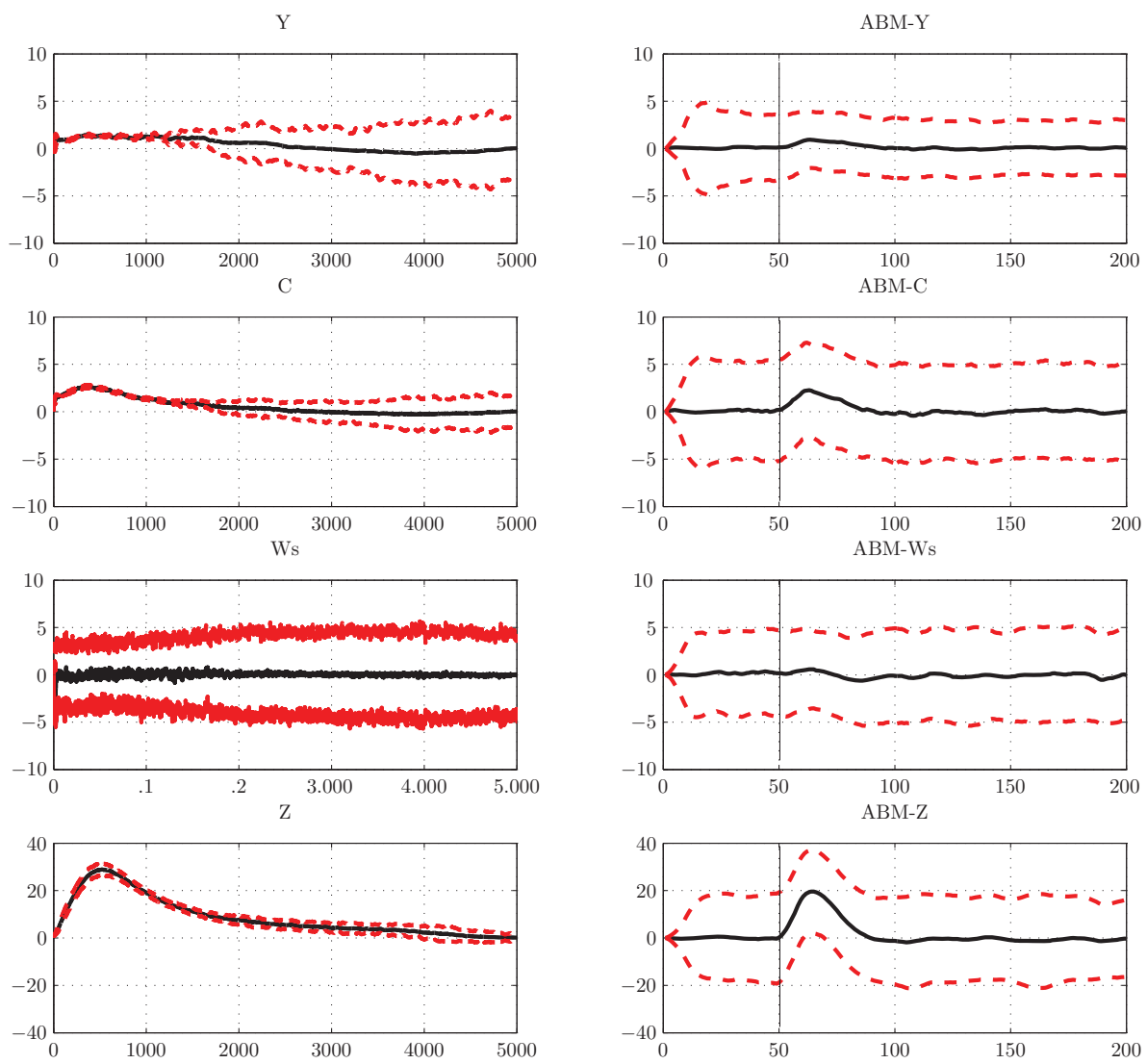

Figure 6: Shock in interest rate $(+1 \%)$. Monte Carlo simulation with 1000 replications. Percentage deviation from baseline simulation and confidence intervals. Left panels: DSG-A system; right panels: ABM. 


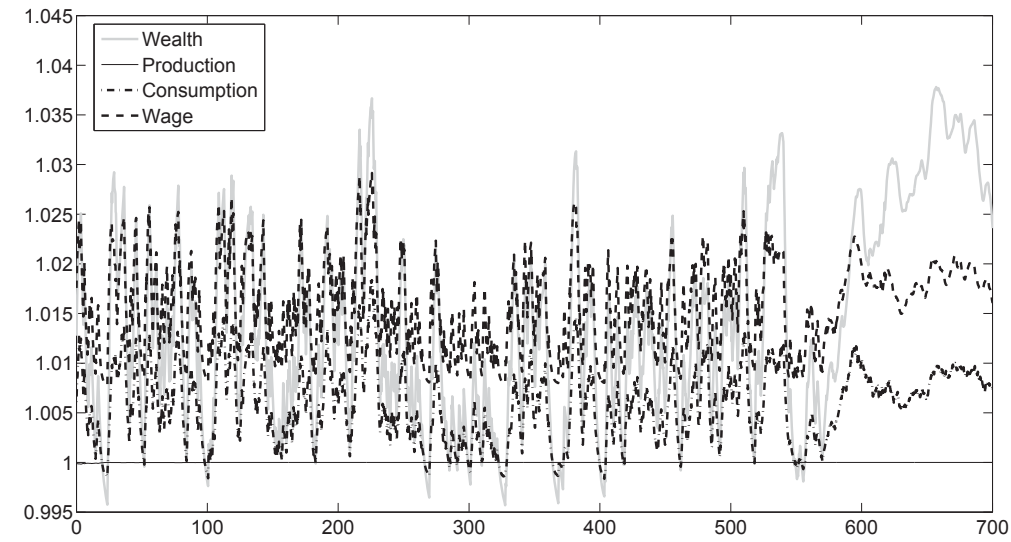

Figure 7: Simulation of the system for the aggregated model with optimization: per-capita real variables (zoom on periods 0-700).

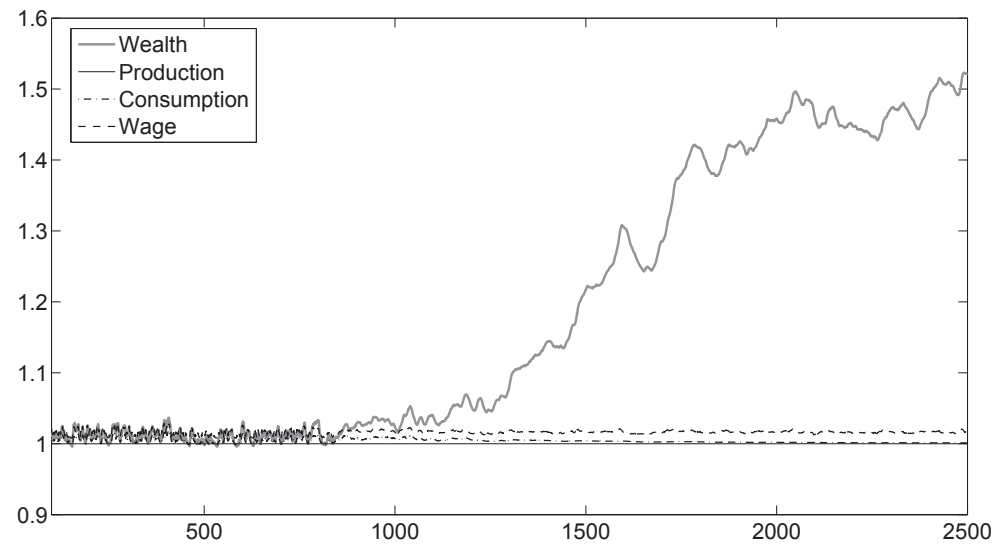

Figure 8: Simulation of the system for the aggregated model with optimization: per-capita real variables (burn-in period 0-100 omitted). 


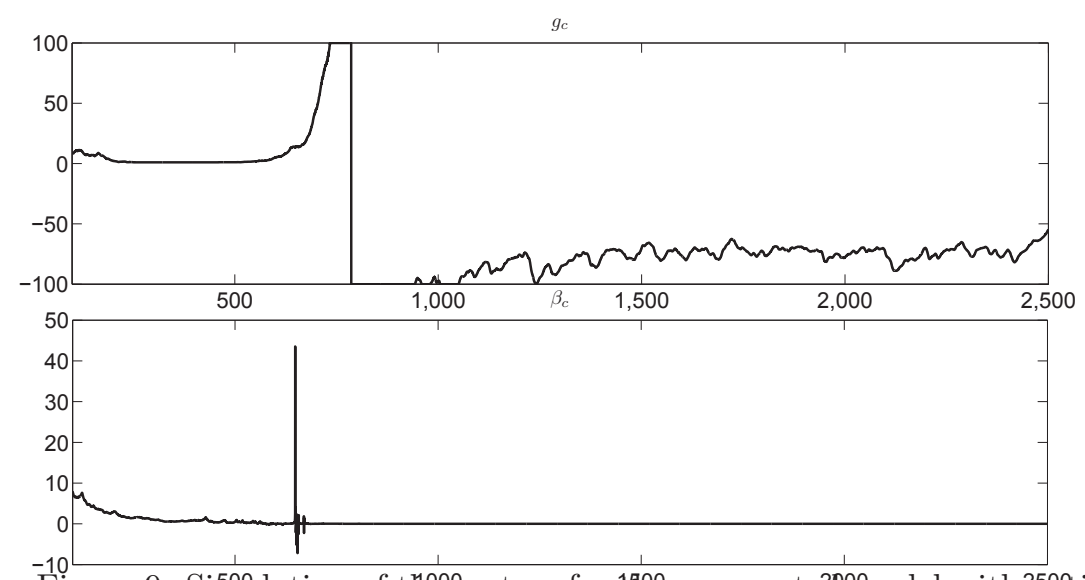

-10 value function $g_{c}$ (upper panel) and uncertainty index $\beta_{c}$ (bottom panel) for supply-constrained consumers (burn-in period 0-100 omitted).

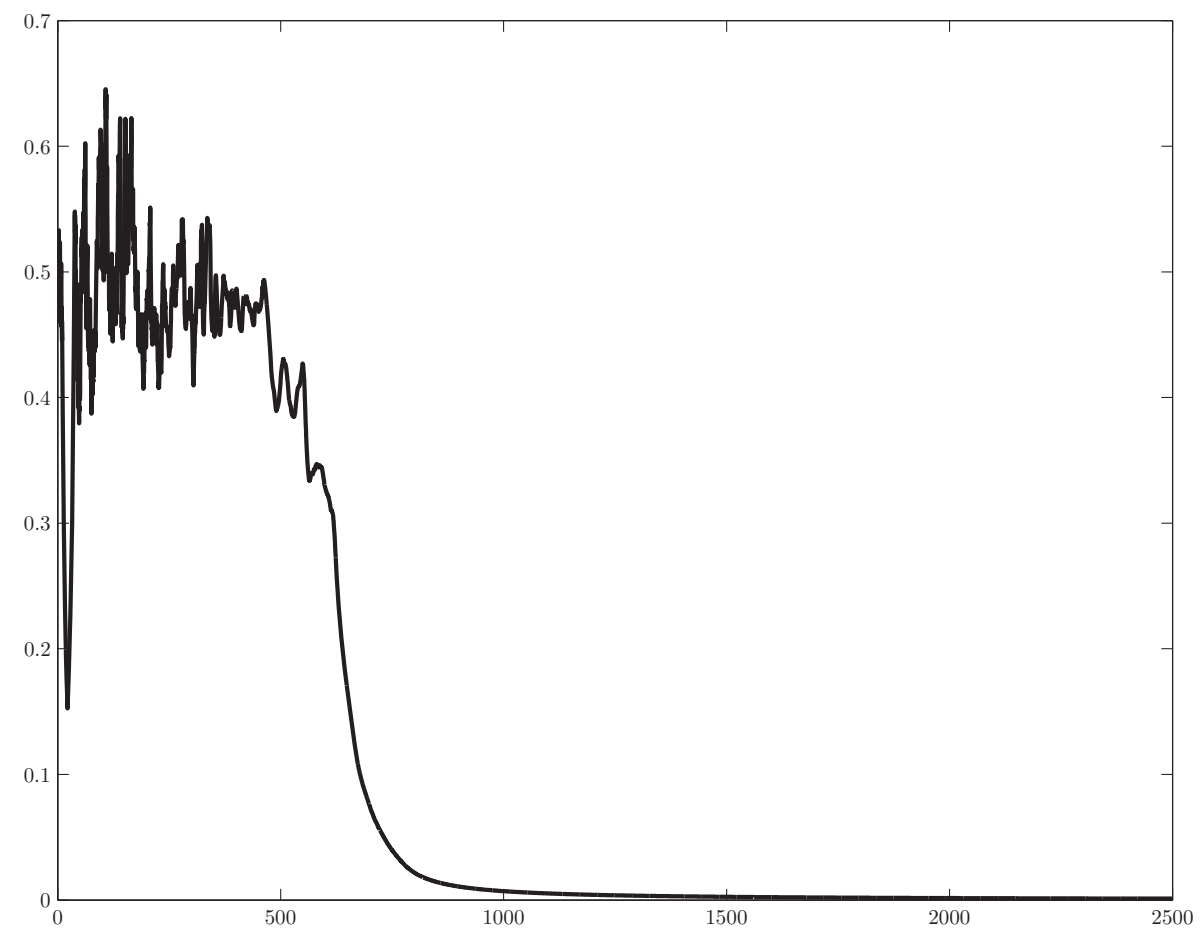

Figure 10: Simulation of the system for the aggregated model with optimization: proportion of households not supply constrained. 


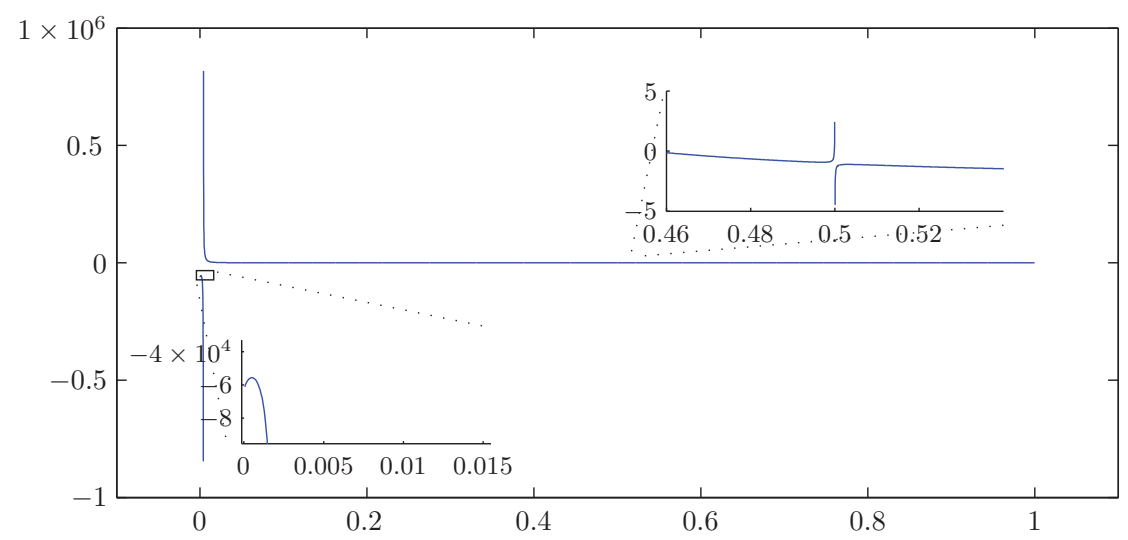

Figure 11: Simulation of the system for the aggregated model with optimization: potential function $U$ (vertical axis) vs. $n_{c}$ (horizontal axis) with zoom around the critical points.
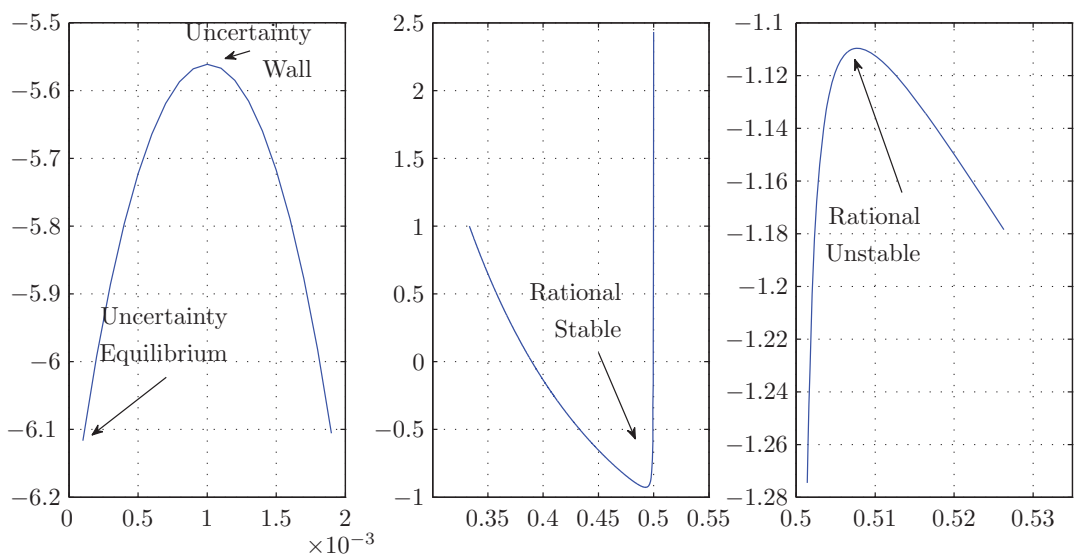

Figure 12: Simulation of the system for the aggregated model with optimization: potential function $U$ (vertical axis) vs. $n_{c}$ (horizontal axis). Detail around the critical points. 

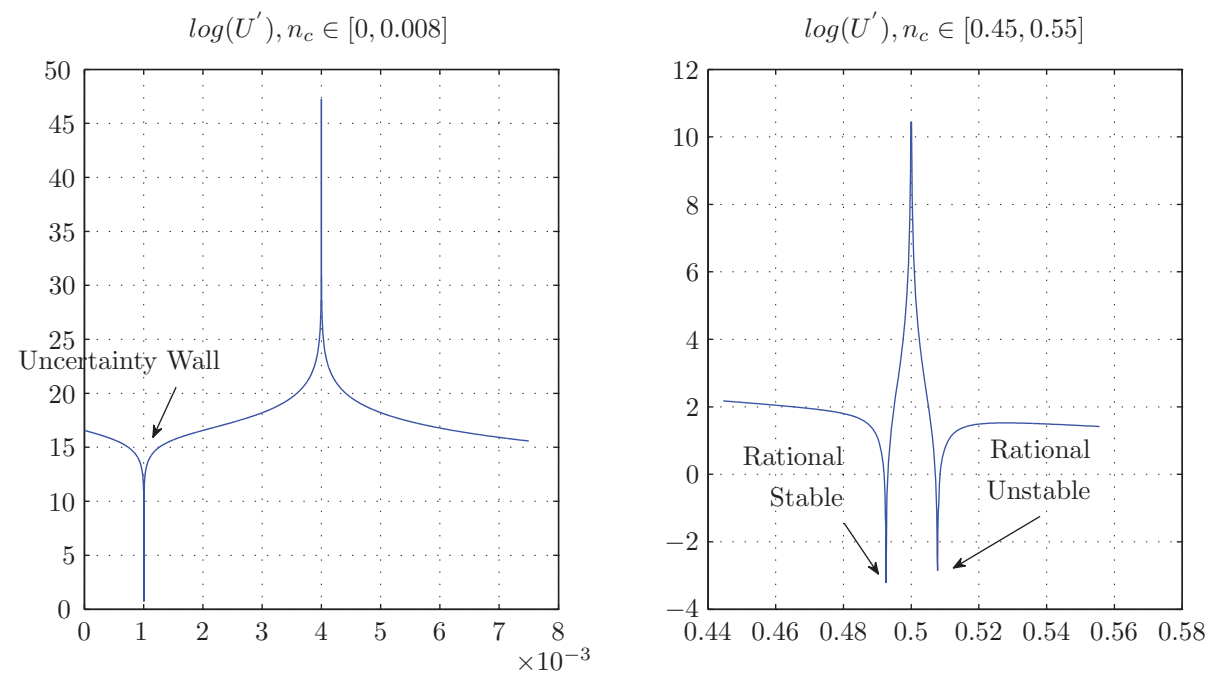

Figure 13: Simulation of the system for the aggregated model with optimization: logarithm of the potential function $U$ (vertical axis) vs. logarithm of $n_{c}$ (horizontal axis). Detail around the critical points.
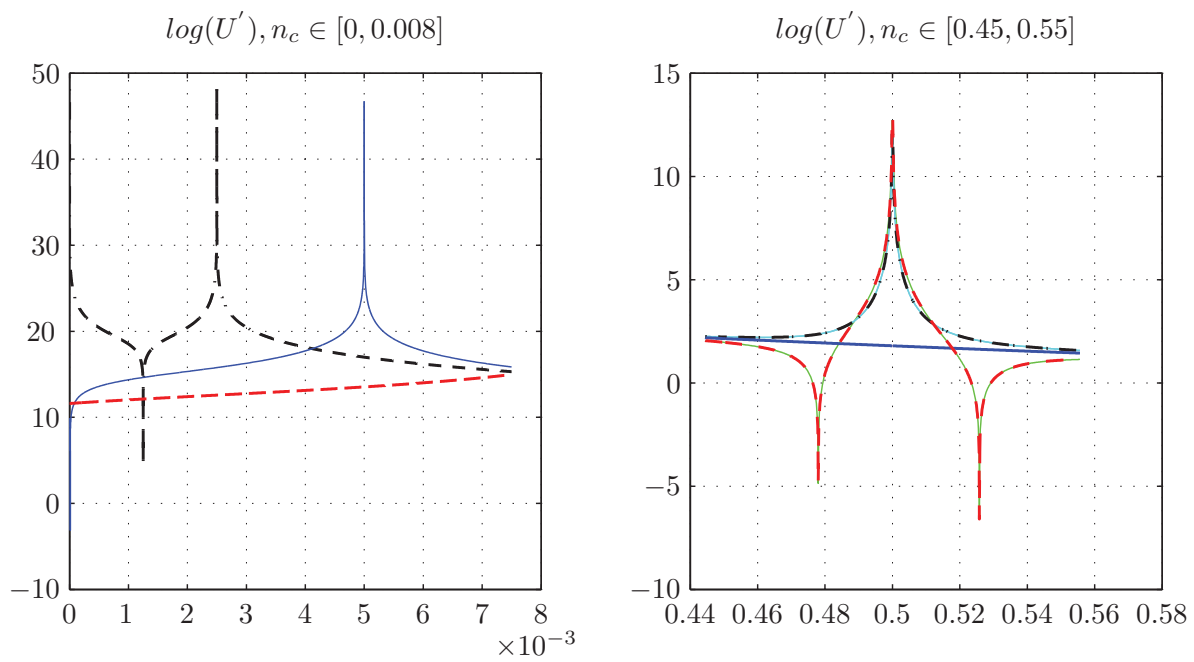

Figure 14: Simulation of the system for the aggregated model with optimization: logarithm of the potential function $U$ (vertical axis) vs. logarithm of $n_{c}$ (horizontal axis). Detail around the critical points for different interest rates. Red line: $r=-0.5$ per cent, black line $r=0.5$ per cent, blue line $r=0$ per cent. 


\section{References}

Alfarano, S., Lux, T. and Wagner, F. (2008): Time variation of higher moments in a financial market with heterogeneous agents: An analytical approach, Journal of Economic Dynamics and Control, 32(1): pp. $101-136$.

Aoki, M. (1996): New approaches to macroeconomic modeling, Cambridge University Press.

Aoki, M. (2002): Modeling aggregate behavior and fluctuations in economics : stochastic views of interacting agents, Cambridge Univ. Press.

Aoki, M. and Yoshikawa, H. (2006): Reconstructing Macroeconomics, Cambridge University Press.

Brock, W. A. and Durlauf, S. N. (2001): Discrete Choice with Social Interactions, Review of Economic Studies, 68(2): pp. 235-60.

Brock, W. A. and Hommes, C. H. (1997): A Rational Route to Randomness, Econometrica, 65(5): pp. 1059-1096.

Chiarella, C. and Di Guilmi, C. (2011): The Financial Instability Hypothesis: A Stochastic Microfoundation Framework, Journal of Economic Dynamics and Control, 35(8): pp. $1151-1171$.

Chiarella, C. and Di Guilmi, C. (2014): The limit distribution of evolving strategies in financial markets, Studies in Nonlinear Dynamics and Econometrics, doi:10.1515/snde-2014-0009, in press.

Clifford, P. (1990): Markov random fields in statistics, in: Grimmett, G. R. and Welsh, D. J. A. (eds.), Disorder in Physical Systems. A Volume in Honour of John M. Hammersley, pp. 19-32, Oxford: Clarendon Press.

Delli Gatti, D., Di Guilmi, C., Gaffeo, E., Giulioni, G., Gallegati, M. and Palestrini, A. (2005): A New Approach to Business Fluctuations: Heterogeneous Interacting Agents, Scaling Laws and Financial Fragility, Journal of Economic Behavior \& Organization, 56(4): pp. 489-512.

Di Guilmi, C. (2008): The Generation of Business Fluctuations: Financial Fragility and Mean-field Interactions, Peter Lang Publishing.

Di Guilmi, C. and Carvalho, L. (2015): The Dynamics of Leverage in a Minskyan Model with Heterogeneous Firms, Working Paper 28, Economics Discipline Group - University of Technology Sydney.

Di Guilmi, C., Gallegati, M., Landini, S. and Stiglitz, J. E. (2012): Dynamic Aggregation of Heterogeneous Interacting Agents and Network: An Analytical Solution for Agent Based Models, Tech. rep., mimeo, URL http://www2.gsb.columbia.edu/faculty/jstiglitz/topics.cfm. 
Dilaver, O., Jump, R. and Levine, P. (2016): Agent-based Macroeconomics and Dynamic Stochastic General Equilibrium Models: Where do we go from here?, Working Paper DP 01/16, University of Surrey, School of Economics.

Dosi, G., Fagiolo, G., Napoletano, M. and Roventini, A. (2013): Income distribution, credit and fiscal policies in an agent-based Keynesian model, Journal of Economic Dynamics and Control, 37(8): pp. 1598-1625.

Foley, D. K. (1994): A Statistical Equilibrium Theory of Markets, Journal of Economic Theory, 62(2): pp. 321-345.

Gardiner, C. W. (2002): Handbook of Stochastic Methods for Physics, Chemistry and the Natural Sciences, Berlin: Springer.

GobBi, A. and Grazzini, J. (2015): Learning and heterogeneity in DSGE models: an agent-based approach, Tech. rep., mimeo.

Izquierdo, L. R., Izquierdo, S. S., Gali, J. M. and Santos, J. I. (2009): Techniques to Understand Computer Simulations: Markov Chain Analysis., Journal of Artificial Societies 65 Social Simulation, 12(1): p. 8.

JAYnes, E. T. (1957): Information Theory and Statistical Mechanics, Phys Rev, 106(4): pp. 620-630, doi:10.1103/PhysRev.106.620.

Journal of Economic Behavior \& Organization (2008): Special issue: Agent-based models for economic policy design, vol. 67,2, editors: Dawid, H. and Fagiolo, G.

Krusell, P., Mukoyama, T., Rogerson, R. and Sahin, A. (2012): Is Labor Supply Important for Business Cycles?, Tech. Rep. 17779, NBER.

Landini, S. and Uberti, M. (2008): A Statistical Mechanics View of Macrodynamics in Economics, Computational Economics, 32(1): pp. 121-146.

Lengnick, M. (2013): Agent-based macroeconomics: A baseline model, Journal of Economic Behavior and Organization, 86(C): pp. 102-120.

Liu, Z. and Phaneuf, L. (2013): The Transmission of Productivity Shocks: What Do We Learn About DSGE Modeling?, Annals of Economics and Statistics, (109-110): pp. 283-304.

Lux, T. (1995): Herd Behaviour, Bubbles and Crashes, Economic Journal, 105(431): pp. 881-96.

Lux, T. (1998): The socio-economic dynamics of speculative markets: interacting agents, chaos, and the fat tails of return distributions, Journal of Economic Behavior 85 Organization, 33(2): pp. 143-165.

Lux, T. (2009): Rational forecasts or social opinion dynamics? Identification of interaction effects in a business climate survey, Journal of Economic Behavior \& Organization, 72(2): pp. $638-655$. 
Russo, A., Catalano, M., Gaffeo, E., Gallegati, M. and Napoletano, M. (2007): Industrial dynamics, fiscal policy and R\&D: Evidence from a computational experiment, Journal of Economic Behavior \& Organization, 64(3-4): pp. 426-447.

Smith, E. and Foley, D. K. (2008): Classical thermodynamics and economic general equilibrium theory, Journal of Economic Dynamics and Control, 32(1): pp. 7-65.

Trigari, A. (2006): The Role of Search Frictions and Bargaining for Inflation Dynamics, Working Papers 304, IGIER (Innocenzo Gasparini Institute for Economic Research), Bocconi University.

VAN KAMPEN, N. G. (1992): Stochastic processes in physics and chemistry., North-Holland, Amsterdam.

Weidlich, W. (2000): Sociodynamics: A Systematic Approach to Mathematical Modelling in the Social Sciences, Gordon and Breach: London.

WeIDLICH, W. (2008): Dynamics of political opinion formation including catastrophe theory, Journal of Economic Behavior \& Organization, 67(1): pp. 1 26. 